\title{
Geometry of orbits of permanents and determinants
}

\author{
Shrawan Kumar
}

\begin{abstract}
We prove that the orbit closure of the determinant is not normal. A similar result is obtained for the padded permanent (i.e., the permanent multiplied by a power of a linear form).
\end{abstract}

Mathematics Subject Classification (2010). 14L30, 14L24, 14R20, 20 G05.

Keywords. Determinant, permanent, normality, geometric complexity, orbit closure.

\section{Introduction}

Let $\mathfrak{v}$ be a complex vector space of dimension $m$ and let $E:=\mathfrak{v} \otimes \mathfrak{v}^{*}=$ End $\mathfrak{v}$. Consider det $\in Q:=S^{m}\left(E^{*}\right)$, where det is the function taking determinant of any $X \in$ End $\mathfrak{v}$. Fix a basis $\left\{e_{1}, \ldots, e_{m}\right\}$ of $\mathfrak{v}$ and a positive integer $n<m$ and consider the function $\mathrm{p} \in Q$, defined by $\mathrm{p}(X)=x_{1,1}^{m-n} \operatorname{perm}\left(X^{o}\right), X^{o}$ being the component of $X$ in the right down $n \times n$ corner, where any element of End $\mathfrak{v}$ is represented by a $m \times m$-matrix $X=\left(x_{i, j}\right)_{1 \leq i, j, \leq m}$ in the basis $\left\{e_{i}\right\}$ and perm denotes the permanent. The group $G=\operatorname{GL}(E)$ canonically acts on $Q$. Let $\mathcal{X}_{\text {det }}$ (resp. $\mathcal{X}_{\mathrm{p}}$ ) be the $G$-orbit closure of det (resp. p) inside $Q$. Then, $\mathcal{X}_{\text {det }}$ and $\mathcal{X}_{\mathrm{p}}$ are closed (affine) subvarieties of $Q$ which are stable under the standard homothety action of $\mathbb{C}^{*}$ on $Q$. Thus, their affine coordinate rings $\mathbb{C}\left[\mathcal{X}_{\text {det }}\right]$ and $\mathbb{C}\left[\mathcal{X}_{\mathrm{p}}\right]$ are nonnegatively graded $G$-algebras over the complex numbers $\mathbb{C}$. Clearly, End $E \cdot \operatorname{det} \subset \mathcal{X}_{\text {det }}$, where End $E$ acts on $Q$ via $(g \cdot q)(X)=q\left(g^{t} \cdot X\right)$ for $g \in$ End $E, q \in Q$ and $X \in E$.

For any positive integer $n$, let $\bar{m}=\bar{m}(n)$ be the smallest positive integer such that the permanent of any $n \times n$ matrix can be realized as a linear projection of the determinant of a $\bar{m} \times \bar{m}$ matrix. This is equivalent to saying that $\mathrm{p} \in$ End $E \cdot \operatorname{det}$ for the pair $(\bar{m}, n)$. Then, Valiant conjectured that the function $\bar{m}(n)$ grows faster than any polynomial in $n$ (cf. [V]).

Similarly, let $m=m(n)$ be the smallest integer such that $\mathrm{p} \in \mathcal{X}_{\text {det }}$ (for the pair $(m, n))$. Clearly, $m(n) \leq \bar{m}(n)$. Now, Mulmuley-Sohoni strengthened Valiant's conjecture. They conjectured that, in fact, the function $m(n)$ grows faster than any polynomial in $n$ (cf. [MS1], [MS2] and the references therein). They further conjectured that if $\mathrm{p} \notin \mathcal{X}_{\mathrm{det}}$, then there exists an irreducible $G$-module which occurs 
in $\mathbb{C}\left[\mathcal{X}_{\mathrm{p}}\right]$ but does not occur in $\mathbb{C}\left[\mathcal{X}_{\text {det }}\right]$. (Of course, if $\mathrm{p} \in \mathcal{X}_{\text {det }}$, then $\mathbb{C}\left[\mathcal{X}_{\mathrm{p}}\right]$ is a $G$-module quotient of $\mathbb{C}\left[\mathcal{X}_{\text {det }}\right]$.) This Geometric Complexity Theory programme initiated by Mulmuley-Sohoni provides a significant mathematical approach to solving the Valiant's conjecture (in fact, strengthened version of Valiant's conjecture proposed by them). In a recent paper, Landsberg-Manivel-Ressayre [LMR] have shown that $m(n) \geq n^{2} / 2$.

It may be remarked that Valiant's above conjecture is equivalent to

$$
\left(\operatorname{perm}_{n}\right)_{n \geq 1} \notin \mathbf{V} \mathbf{P}_{\text {ws }} .
$$

This is an algebraic version of Cook's celebrated $\mathbf{P} \neq \mathbf{N P}$ conjecture. The conjecture of Mulmuley-Sohoni is equivalent to $\left(\text { perm }_{n}\right)_{n \geq 1} \notin \overline{\mathbf{V P}_{\mathbf{w s}}}$. For a survey of these problems, we refer to the article [BL] by Bürgisser-Landsberg-Manivel-Weyman.

From the experience in representation theory (e.g., the Demazure character formula or the study of functions on the nilpotent cone), one important property of varieties which allows one to study the ring of regular functions on them is their normality. But, unfortunately, as we show in the paper, both of the varieties $\mathcal{X}_{\text {det }}$ (for any $m \geq 3$ ) and $X_{\mathrm{p}}$ (for any $m \geq n+1$ and $n \geq 3$ ) are not normal (cf. Theorems 3.8 and 8.4). These are the principal results of the paper.

To prove the nonnormality of $\mathcal{X}_{\mathrm{det}}$, we study the defining equations of the boundary $\partial \mathcal{X}_{\mathrm{det}}:=\mathcal{X}_{\mathrm{det}} \backslash \mathcal{X}_{\mathrm{det}}^{o}$ and show that there exists a $G^{\prime}$-invariant $f_{o}$ in $\mathbb{C}\left[\mathcal{X}_{\mathrm{det}}\right]$ (where $G^{\prime}:=\operatorname{SL}(E)$ and $X_{\text {det }}^{o}:=G \cdot \operatorname{det}$ ), which defines $\partial X_{\text {det }}$ set theoretically (but not scheme theoretically), cf. Corollaries 3.6 and 3.9. In particular, each irreducible component of $\partial \mathcal{X}_{\text {det }}$ is of codimension one in $\mathcal{X}_{\text {det }}$ (cf. Corollary 3.6). To show that $\mathcal{X}_{\mathrm{det}}$ is not normal, we show that, in fact, the GIT quotient $\mathcal{X}_{\mathrm{det}}^{\prime}:=\mathcal{X}_{\mathrm{det}} / / G^{\prime}$ is not normal by analyzing the $G^{\prime}$-invariants in $\mathbb{C}\left[\mathcal{X}_{\text {det }}\right]$.

Let $\left\{e_{1}^{*}, \ldots, e_{m}^{*}\right\}$ be the dual basis of $\mathfrak{v}^{*}$. Then, of course, $\left\{e_{i, j}:=e_{i} \otimes e_{j}^{*} ; 1 \leq\right.$ $i, j \leq m\}$ is a basis of $E$. Let $S_{1}$ be the subspace of $E$ spanned by $\left\{e_{i, j} ; m-n+1 \leq\right.$ $i, j \leq m\}, S$ the subspace of $E$ spanned by $S_{1}$ and $e_{1,1}$, and $S^{\perp}$ the complementary subspace spanned by the set $\left\{e_{i, j}\right\}_{1 \leq i, j, \leq m} \backslash\left\{e_{1,1}, e_{i, j}\right\}_{m-n+1 \leq i, j \leq m}$. Let $P$ be the maximal parabolic subgroup of $G=\mathrm{GL}(E)$ which keeps the subspace $S^{\perp}$ of $E$ stable and let $L_{P}$ be the Levi subgroup of $P$ defined by $L_{P}=\operatorname{GL}\left(S^{\perp}\right) \times \operatorname{GL}(S)$. Let $R$ be the parabolic subgroup of $\operatorname{GL}(S)$ which fixes the line spanned by $e_{1,1}$.

The proof of the nonnormality of $\mathcal{X}_{\mathrm{p}}$ is more involved. We first show that the $G$-module decomposition of $\mathbb{C}\left[\mathcal{X}_{\mathrm{p}}\right]$ is equivalent to the $\operatorname{GL}(S)$-module decomposition of the ring of the regular functions on the GL(S)-orbit closure $\mathcal{C}$ of $\mathrm{p}$ (cf. Theorem 5.2). Next, we analyze $\mathcal{C}$ in Section 6. In particular, we give its partial desingularization of the form $\mathscr{D}:=\operatorname{GL}(S) \times_{R}\left(\left(S^{*} \times \mathcal{X}_{\text {perm }}\right) / / \mathbb{C}^{*}\right)$ (cf. Proposition 6.3 and Lemma 6.2), where $\mathcal{X}_{\text {perm }}$ is the GL( $\left.S_{1}\right)$-orbit closure of the permanent function perm inside $S^{n}\left(E^{*}\right), \mathbb{C}^{*}$ acts on $S^{*} \times \mathcal{X}_{\text {perm }}$ via the equation (21) and the action of $R$ on $\left(S^{*} \times \mathcal{X}_{\text {perm }}\right) / / \mathbb{C}^{*}$ is given in Section 6 immediately after Lemma 6.2. We determine the ring of regular functions on $\mathscr{D}$ (as a GL(S)-module) completely 
(and explicitly) in terms of the ring of regular functions on $\mathcal{X}_{\text {perm }}$ as a $\operatorname{GL}\left(S_{1}\right)$ module (cf. Theorem 7.5). Via the Zariski's main theorem, this allows one to give the $G$-module decomposition of the normalization of $\mathcal{X}_{\mathrm{p}}$ completely in terms of the $\mathrm{GL}\left(S_{1}\right)$-module decomposition of the ring of regular functions on the normalization of the GL( $\left(S_{1}\right)$-variety $\mathcal{X}_{\text {perm }}$ (use Theorem 5.2, Corollary 5.4, Lemma 6.2, Proposition 6.3 and Theorem 7.5). It may be remarked that we are not able to give an explicit $G$-module decomposition of $\mathbb{C}\left[\mathcal{X}_{\mathrm{p}}\right]$ itself from that of the $\operatorname{GL}\left(S_{1}\right)$-module $\mathbb{C}\left[\mathcal{X}_{\text {perm }}\right]$. By comparing the explicit $\operatorname{GL}(S)$-module decomposition of the ring of regular functions $\mathbb{C}[D]$ mentioned above with the ring of regular functions on the $\mathrm{GL}(S)$-orbit closure of $\mathrm{p}$, we conclude that $\mathcal{X}_{\mathrm{p}}$ is not normal for any $m \geq n+1$ and $n \geq 3$ (cf. Theorem 8.4). A similar idea allows us to conclude that the orbit closures of $\mathrm{p}$ under the groups $R$ and GL(S) are not normal (cf. Corollaries 8.2 and 8.3).

Notation. We have often abused the notation and denoted the homogeneous vector bundle on the homogeneous space $G / P$ associated to the $P$-module $M$ by $M$ itself. Hopefully, the distinction will be clear from the context. We denote $\mathbb{C} \backslash\{0\}$ by $\mathbb{C}^{*}$ and the dual of a vector space $V$ by $V^{*}$. (We hope it will not cause any confusion.)

Acknowledgements. I thank J. Landsberg for bringing my attention to the works of Mulmuley-Sohoni and his comments to an earlier version of the paper and to K. Mulmuley for explaining to me some of his works. I thank the referee for some helpful comments. This work was partially supported by the NSF grant DMS 0901239.

\section{Coordinate ring of the orbit closure of det}

Take a vector space $\mathfrak{v}$ of dimension $m>0$ and let $E=\mathfrak{v} \otimes \mathfrak{v}^{*}=$ End $\mathfrak{v}$. Consider $G=\mathrm{GL}(E)$ acting canonically on $Q=S^{m}\left(E^{*}\right)$, and consider det $\in Q$, where det is the function taking determinant of any $A \in$ End $\mathfrak{v}$.

Recall the following result due to Frobenius [Fr] (cf., e.g., [GM] for a survey).

2.1 Proposition. The isotropy $G_{\mathrm{det}} \subset G$ consists of the transformations of the form $\tau: Y \mapsto A Y^{*} B$, where $Y^{*}=Y$ or $Y^{t}$ and $A, B \in \mathrm{SL}(\mathfrak{v})$. (Here $Y^{t}$ denotes the transpose of $Y$ with respect to a fixed basis of $\mathfrak{w}$.)

2.2 Lemma. Any $\tau$ of the form $\tau(Y)=A Y B$ as above can be written as

$$
\text { End } \mathfrak{v}=\mathfrak{v} \otimes \mathfrak{v}^{*} \rightarrow \mathfrak{v} \otimes \mathfrak{v}^{*}, \quad v \otimes f \mapsto A v \otimes B^{*} f,
$$

where $B^{*}$ is the dual map induced from B. In particular, such a $\tau$ has determinant 1 .

If $\tau$ is of the form $\tau(Y)=A Y^{t} B$ as in the above proposition, then

$$
\operatorname{det} \tau=(-1)^{\frac{m(m-1)}{2}} \text {. }
$$


Proof. Take a basis $\left\{e_{i}\right\}$ of $\mathfrak{v}$ and let $\left\{e_{i}^{*}\right\}$ be the dual basis of $\mathfrak{v}^{*}$. Let $A=\left(a_{i, j}\right)$ be the matrix of $A$ in the basis $\left\{e_{i}\right\}$ of $\mathfrak{v}$ and similarly $B=\left(b_{i, j}\right)$. Then,

$$
\left(B^{*} e_{j}^{*}\right) e_{p}=e_{j}^{*}\left(B e_{p}\right)=\sum_{\ell} e_{j}^{*}\left(b_{\ell, p} e_{\ell}\right)=b_{j, p} .
$$

Thus, $B^{*} e_{j}^{*}=\sum_{p} b_{j, p} e_{p}^{*}$. Hence, denoting the map (1) by $\hat{\tau}$, we have

$$
e_{i, j}:=e_{i} \otimes e_{j}^{*} \stackrel{\hat{\tau}}{\longmapsto} A e_{i} \otimes B^{*}\left(e_{j}^{*}\right)=\sum_{k, p} a_{k, i} e_{k} \otimes b_{j, p} e_{p}^{*}=\sum_{k, p} a_{k, i} b_{j, p} e_{k} \otimes e_{p}^{*} .
$$

Thus,

$$
\left(\hat{\tau}\left(e_{i, j}\right)\right)_{k, p}=a_{k, i} b_{j, p}=\left(A e_{i, j} B\right)_{k, p},
$$

where $\left(\hat{\tau}\left(e_{i, j}\right)\right)_{k, p}$ denotes the $(k, p)$-th component of $\hat{\tau}\left(e_{i, j}\right)$ in the basis $\left\{e_{k, p}\right\}$. This proves $\tau=\hat{\tau}$.

Let $\left\{\lambda_{1}, \ldots, \lambda_{m}\right\}$ be the eigenvalues of $A$ and $\left\{\mu_{1}, \ldots, \mu_{m}\right\}$ the eigenvalues of $B$. Then,

$$
\operatorname{det} \hat{\tau}=\prod_{i, j=1}^{m} \lambda_{i} \mu_{j}=\prod_{i}\left(\lambda_{i}^{m} \operatorname{det} B\right)=(\operatorname{det} A)^{m}(\operatorname{det} B)^{m}=1,
$$

since $\operatorname{det} A=\operatorname{det} B=1$.

To prove (2), in view of the above, we can assume that $\tau(Y)=Y^{t}$. The proof in this case is easy.

As a consequence of Proposition 2.1 and Lemma 2.2, we get the following.

2.3 Corollary. We have a group isomorphism:

$$
\phi: \operatorname{SL}(\mathfrak{v}) \times \mathrm{SL}(\mathfrak{v}) / \Theta_{m} \simeq G_{\mathrm{det}}^{o}, \quad \phi[A, B](v \otimes f)=A v \otimes\left(B^{-1}\right)^{*} f,
$$

where $\Theta_{m}$ is the group of the $m$-th roots of unity acting on $\mathrm{SL}(\mathfrak{v}) \times \mathrm{SL}(\mathfrak{y})$ via $z(A, B)=(z A, z B),[A, B]$ denotes the $\Theta_{m}$-orbit of $(A, B)$ and $G_{\mathrm{det}}^{o}$ denotes the identity component of $G_{\mathrm{det}}$.

In particular, $\operatorname{dim}\left(G^{\prime} \cdot \operatorname{det}\right)=\left(m^{2}-1\right)^{2}$, where $G^{\prime}:=\operatorname{SL}(E)$. Moreover, $G_{\mathrm{det}}^{o} \subset G_{\mathrm{det}}^{\prime}$.

If $\left(\begin{array}{c}m \\ 2\end{array}\right)$ is even, then $G_{\mathrm{det}} \subset G^{\prime}$.

Since the isotropy $G_{\mathrm{det}}^{\prime}$ is not contained in any proper parabolic subgroup of $G^{\prime}$ (as can be easily seen by observing that no proper subspace of $E$ is stable under $G_{\text {det }}^{o}$ ), Kempf's theorem [Ke], Corollary 5.1, gives the following result observed in Theorem 4.6 of [MS1]: 
2.4 Proposition. The orbit $G^{\prime} \cdot \operatorname{det}$ is closed in $Q$.

Let $\mathcal{X}_{\mathrm{det}}^{o}:=G \cdot \operatorname{det}, \mathcal{X}_{\mathrm{det}}:=\overline{X_{\mathrm{det}}^{o}}$, where the closure is taken inside $Q$, and let $X_{\text {det }}^{\prime}:=G^{\prime} \cdot$ det. More generally, let $V$ be an irreducible representation of GL $(k)$ (for some $k \geq 1)$ such that the center of $\operatorname{GL}(k)$ acts nontrivially on $V$ and let $v_{o} \in V$ be such that $\operatorname{SL}(k)$-orbit of $v_{o}$ is closed. Denote $X=\overline{\mathrm{GL}(k) \cdot v_{o}}$ and $X^{\prime}=\mathrm{SL}(k) \cdot v_{o}$. The following simple lemma is taken from [MS2].

2.5 Lemma. For any $d \geq 0$, the restriction map

$$
\phi^{d}: \mathbb{C}^{d}[X] \rightarrow \mathbb{C}\left[X^{\prime}\right]
$$

is injective, where $\mathbb{C}^{d}[X]$ is the homogeneous degree $d$-part of $\mathbb{C}[X]$ (i.e., $\mathbb{C}^{d}[X]$ is a quotient of $\left.S^{d}\left(V^{*}\right)\right)$.

In particular, for any $d \geq 0$, the restriction map

$$
\phi^{d}: \mathbb{C}^{d}\left[\mathcal{X}_{\mathrm{det}}\right] \rightarrow \mathbb{C}\left[\mathcal{X}_{\mathrm{det}}^{\prime}\right]
$$

is injective.

Proof. Take $f \in \mathbb{C}^{d}[X]$ such that $\phi^{d}(f)=0$, i.e., $f(x)=0$ for all $x \in X^{\prime}$. Then, for any $z \in \mathbb{C}$ and $x \in X^{\prime}, f(z x)=z^{d} f(x)=0$, i.e., $f\left(\mathbb{C} \cdot X^{\prime}\right) \equiv 0$ and hence $f\left(\overline{\mathbb{C} \cdot X^{\prime}}\right) \equiv 0$. But, $\overline{\mathbb{C} \cdot X^{\prime}}=X$ and hence $f(X) \equiv 0$. This proves the lemma.

As a consequence of Proposition 2.4, Lemma 2.5 and the Frobenius reciprocity, one has the following result from [MS2].

2.6 Corollary. An irreducible $G^{\prime}$-module $M$ occurs in $\mathbb{C}\left[G^{\prime} / G_{\mathrm{det}}^{\prime}\right]=\mathbb{C}\left[\mathcal{X}_{\mathrm{det}}^{\prime}\right]$ if and only if $M$ occurs in $\mathbb{C}\left[\mathcal{X}_{\text {det }}\right]$. In particular, an irreducible $G^{\prime}$-module $M$ occurs in $\mathbb{C}\left[\mathcal{X}_{\mathrm{det}}\right]$ if and only if $M^{G_{\mathrm{det}}^{\prime}} \neq 0$.

2.7 Example. Let $m=2$. Then, $G$. det is dense in $Q=S^{2}\left(E^{*}\right)$ (since they have the same dimensions by Corollary 2.3). Moreover, $Q$ has 5 orbits under $G$ of dimensions: $10,9,7,4,0$.

To show this, observe that there are exactly 5 quadratic forms in 4 variables (up to the change of a basis): $x_{1}^{2}+x_{2}^{2}+x_{3}^{2}+x_{4}^{2} ; x_{1}^{2}+x_{2}^{2}+x_{3}^{2} ; x_{1}^{2}+x_{2}^{2} ; x_{1}^{2} ; 0$. Their isotropies under the $G$-action have dimensions: $6,7,9,12,16$ respectively.

\section{Non-normality of the orbit closure of det}

We first recall the following two elementary lemmas from commutative algebra. 
3.1 Lemma. Let $R$ be a $\mathbb{Z}_{+}$-graded algebra over the complex numbers $\mathbb{C}$ with the degree 0 -component $R^{0}=\mathbb{C}$ and let $M$ be a $\mathbb{Z}_{+}$-graded $R$-module. Let $\mathrm{m}$ be the augmentation ideal $\bigoplus_{d>0} R^{d}$ and assume that $M /(\mathfrak{m} \cdot M)$ is a finite dimensional vector space over $R / \mathfrak{m} \simeq \mathbb{C}$. Then, $M$ is a finitely generated $R$-module.

Proof. Choose a set of homogeneous generators $\left\{\bar{x}_{1}, \ldots, \bar{x}_{n}\right\} \subset M /(\mathfrak{m} \cdot M)$ over $R / \mathfrak{m}$ and let $x_{i} \in M$ be a homogeneous lift of $\bar{x}_{i}$. Let $N \subset M$ be the graded $R$-submodule: $R x_{1}+\cdots+R x_{n}$. It is easy to see that

$$
\mathfrak{m} \cdot(M / N)=M / N \text {. }
$$

If $M / N \neq 0$, let $d_{o} \geq 0$ be the smallest degree such that $(M / N)^{d_{o}} \neq 0$. Clearly, (3) contradicts this. Hence $N=M$.

3.2 Lemma. Let $R$ and $S$ be two non-negatively graded finitely generated domains over $\mathbb{C}$ such that $R^{0}=S^{0}=\mathbb{C}$ and let $f: R \rightarrow S$ be a graded algebra injective homomorphism. Assume that the induced map $\hat{f}: \operatorname{Spec} S \rightarrow \operatorname{Spec} R$ satisfies $(\hat{f})^{-1}\left(\mathfrak{m}_{R}\right)=\left\{\mathfrak{m}_{S}\right\}$, where $\mathfrak{m}_{S}$ is the augmentation ideal of $S$ and $\operatorname{Spec} S$ denotes the space of maximal ideals of $S$. Then, $S$ is a finitely generated $R$-module; in particular, it is integral over $R$.

Proof. Let $\mathfrak{m}_{R}^{\prime}$ be the ideal in $S$ generated by $f\left(\mathfrak{m}_{R}\right)$. Then, by assumption, $\mathfrak{m}_{S}$ is the only maximal ideal of $S$ containing $\mathfrak{m}_{R}^{\prime}$. Hence, the radical ideal $\sqrt{ } \mathfrak{m}_{R}^{\prime}=\mathfrak{m}_{S}$. Thus, $\mathfrak{m}_{R}^{\prime} \supset \mathfrak{m}_{S}^{d}$ for some $d>0$ (cf. [AM], Corollary 7.16). In particular, $S / \mathfrak{m}_{R}^{\prime}$ is a finite dimensional vector space over $\mathbb{C}$ and hence by the above lemma, $S$ is a finitely generated $R$-module. This proves that $S$ is integral over $R$ (cf. [AM], Proposition 5.1).

Let $\partial \mathcal{X}_{\text {det }}:=\mathcal{X}_{\operatorname{det}} \backslash \mathcal{X}_{\text {det }}^{o}$ be its boundary, equipped with the closed (reduced) subvariety structure coming from $Q$. Let $\mathcal{I} \subset \mathbb{C}\left[\mathcal{X}_{\mathrm{det}}\right]$ denote the ideal of $\partial \mathcal{X}_{\mathrm{det}}$. More generally, as in Lemma 2.5, let $V$ be an irreducible representation of GL $(k)$ (for some $k \geq 1)$ such that the center of $\mathrm{GL}(k)$ acts nontrivially on $V$ and let $0 \neq v_{o} \in V$ be such that $\mathrm{SL}(k)$-orbit of $v_{o}$ is closed. Denote $X^{o}=\mathrm{GL}(k) \cdot v_{o}, X=\overline{\mathrm{GL}}(k) \cdot v_{o}$ and $\partial X=X \backslash X^{o}$, all equipped with the locally-closed (reduced) subvariety structures coming from that of $V$. Let $I \subset \mathbb{C}[X]$ denote the ideal of $\partial X$. With this notation, we have the following Lemma 3.3, Proposition 3.5 and Corollary 3.6.

3.3 Lemma. For any nonzero GL( $(k)$-submodule $M \subset I$, the zero set

$$
Z(M):=\{y \in X: f(y)=0 \text { for all } f \in M\}
$$

equals $\partial X$. 
Proof. Of course, $Z(M) \supset \partial X$. Moreover, $Z(M)$ is a GL $(k)$-stable subset of $X$. If $Z(M)$ properly contains $\partial X$, then $Z(M)=X$, which is a contradiction since $M$ is nonzero.

3.4 Remark. The above lemma is clearly true even without the assumption that $\mathrm{SL}(k) \cdot v_{o}$ is closed.

3.5 Proposition. The ideal $I \subset \mathbb{C}[X]$ contains a nonzero $\mathrm{SL}(k)$-invariant. In particular, the ideal $\mathcal{I} \subset \mathbb{C}\left[\mathcal{X}_{\mathrm{det}}\right]$ contains a nonzero $G^{\prime}$-invariant.

Proof. Let $m_{o}$ be the unique integer such that $\left(z I_{k}\right) \cdot v_{o}=z^{-m_{o}} v_{o}$ for all $z \in \mathbb{C}^{*}$, where $I_{k}$ is the identity matrix in $\operatorname{GL}(k)$. Consider the action of $\mathbb{C}^{*}$ on $V$ via $z \cdot v=\left(z^{\epsilon\left(m_{o}\right)} I_{k}\right) \cdot v$, where

$$
\begin{aligned}
\epsilon\left(m_{o}\right) & =-1 & & \text { if } m_{o}>0 \\
& =1 & & \text { if } m_{o}<0 .
\end{aligned}
$$

This gives rise to an action of $\mathbb{C}^{*}$ on $X$. Let $Z:=X / / \operatorname{SL}(k)$. Then, $Z$ is an irreducible affine variety with $\mathbb{C}^{*}$-action coming from the action of $\mathbb{C}^{*}$ on $X$. Consider the $\mathbb{C}^{*}$-equivariant map $\sigma: \mathbb{C} \rightarrow X, w \mapsto w^{-\epsilon\left(m_{o}\right) m_{o}} v_{o}$, where $\mathbb{C}^{*}$ acts on $\mathbb{C}$ via $z \cdot w=z w$. Consider the composite map $\bar{\sigma}=\pi \circ \sigma: \mathbb{C} \rightarrow Z$, where $\pi: X \rightarrow X / / \operatorname{SL}(k)$ is the canonical projection. By the assumption that $\operatorname{SL}(k) \cdot v_{o}$ is closed in $V,(\bar{\sigma})^{-1}\{0\}=\{0\}$. Moreover, clearly $\bar{\sigma}$ is a dominant morphism since $\mathrm{GL}(k) \cdot v_{o}$ is dense in $X$. Thus, by Lemma 3.2, $\bar{\sigma}$ is a finite (in particular, surjective) morphism. Moreover, no SL( $k)$-orbit $Y$ in $\partial X \backslash\{0\}$ is closed in $X$. In fact, for any such $Y, 0 \in \bar{Y}$ :

Let $Y^{\prime}$ be a closed $\operatorname{SL}(k)$-orbit in $\bar{Y}$. If $Y^{\prime}$ is nonzero, $Y^{\prime}=\operatorname{SL}(k) \cdot \sigma(z)$, for some $z \in \mathbb{C}^{*}$, since $\bar{\sigma}$ is surjective. But, $\operatorname{SL}(k) \cdot \sigma(z) \subset X^{o}$, whereas $Y^{\prime} \subset \partial X$. This is a contradiction. Hence, $0 \in \bar{Y}$.

Take any nonzero homogeneous polynomial $f_{o} \in \mathbb{C}[Z]=\mathbb{C}[X]^{\mathrm{SL}(k)}$ of positive degree. Then, $f_{o}$ restricted to $\partial X / / \mathrm{SL}(k)$ is identically zero, since $\partial X / / \operatorname{SL}(k) \simeq$ $\{0\}$. Hence, $f_{o} \in I$. This proves the proposition.

3.6 Corollary. For any nonzero homogeneous $f_{o} \in \mathbb{C}[X]^{\mathrm{SL}(k)}$ of positive degree, the zero set $Z\left(f_{o}\right)=\partial X$. In particular,

$$
\sqrt{ }\left\langle f_{o}\right\rangle=I,
$$

where $\left\langle f_{o}\right\rangle$ is the ideal of $\mathbb{C}[X]$ generated by $f_{o}$.

Moreover, each irreducible component of $\partial X$ is of codimension one in $X$. In particular, each irreducible component of $\partial \mathcal{X}_{\mathrm{det}}$ is of codimension one in $\mathcal{X}_{\mathrm{det}}$.

Proof. By the last paragraph of the proof of the above proposition, $f_{o \mid \partial X} \equiv 0$. Thus, the first part of the corollary is a particular case of Lemma 3.3. 
For the second part, observe that $f_{o}$ does not vanish anywhere on $X^{o}$ since $f_{o}$ is $\mathrm{SL}(k)$-invariant and homogeneous. Moreover, $f_{o} \circ \bar{\sigma}: \mathbb{C} \rightarrow \mathbb{C}$ is surjective (being nonzero) and hence so is $f_{o}: X \rightarrow \mathbb{C}$. Now use [S], Theorem 7 on page 76.

3.7 Remark. The assertion in the above corollary, that each irreducible component of $\partial X$ is of codimension one in $X$, can also be proved by using Lemma 5.7. (Observe that $\operatorname{GL}(k) \cdot v_{o}$ is affine by using Matsushima's theorem.)

3.8 Theorem. For any $m \geq 3, \mathcal{X}_{\mathrm{det}}=\overline{G \cdot \operatorname{det}}$ is not normal.

Proof. Assume that $\mathcal{X}_{\text {det }}$ is normal, then so would be $Z=X_{\operatorname{det}} / / G^{\prime}$. By Matsushima's theorem, since the isotropy of det is reductive (cf. Corollary 2.3), $\mathcal{X}_{\mathrm{det}}^{o}$ is an affine variety. By the Frobenius reciprocity,

$$
\mathbb{C}\left[\mathcal{X}_{\mathrm{det}}^{o}\right]^{G^{\prime}} \simeq \bigoplus_{a \in \mathbb{Z}} V(a D) \otimes\left[V(a D)^{*}\right]^{G_{\mathrm{det}}},
$$

where $V(a D)$ is the irreducible $G$-module of dimension one with highest weight corresponding to the partition ( $a \geq \cdots \geq a)$ ( $m^{2}$ factors). Thus, $V(a D)$ is the one dimensional representation corresponding to the character $g \mapsto(\operatorname{det} g)^{a}$. By Lemma 2.2, if $m(m-1) / 2$ is even, $\left[V(a D)^{*}\right]^{G_{\text {det }}}$ is one dimensional, for all $a \in \mathbb{Z}$. If $m(m-1) / 2$ is odd,

$$
\begin{aligned}
\operatorname{dim}\left[V(a D)^{*}\right]^{G_{\text {det }}} & =1 & & \text { if } a \text { is even, } \\
& =0 & & \text { if } a \text { is odd }
\end{aligned}
$$

For $d \in \mathbb{Z}_{+}$, let $\mathbb{C}^{d}\left[\mathcal{X}_{\mathrm{det}}^{o}\right]$ denote the subspace of $\mathbb{C}\left[\mathcal{X}_{\mathrm{det}}^{o}\right]$ such that, for any $z \in \mathbb{C}^{*}$, the matrix $z I$ acts via $z^{m d}$. Let $\hat{f}_{o} \in \mathbb{C}^{p_{m} m}\left[\mathcal{X}_{\text {det }}^{o}\right]^{G^{\prime}}$ be a nonzero element, where $p_{m}=1$ if $m(m-1) / 2$ is even and $p_{m}=2$ if $m(m-1) / 2$ is odd. Then, clearly,

$$
\mathbb{C}^{\geq 0}\left[\mathcal{X}_{\mathrm{det}}^{o}\right]^{G^{\prime}} \simeq \bigoplus_{a \in \mathbb{Z}_{+}} \mathbb{C} \hat{f}_{o}^{a} .
$$

Now, $\mathbb{C}\left[\mathcal{X}_{\mathrm{det}}\right]^{G^{\prime}} \subset \mathbb{C}\left[\mathcal{X}_{\mathrm{det}}^{o}\right]^{G^{\prime}}$ is a homogeneous subalgebra. Let $d_{o}>0$ be the smallest integer such that $f_{o}=\hat{f}_{o}^{d_{o}} \in \mathbb{C}\left[\mathcal{X}_{\mathrm{det}}\right]^{G^{\prime}}$. (Such a $d_{o}$ exists by Proposition 3.5.) Since, by assumption, $\mathbb{C}\left[\mathcal{X}_{\mathrm{det}}\right] G^{G^{\prime}}$ is a normal ring, $\hat{f}_{o} \in \mathbb{C}^{p^{m} m}\left[\mathcal{X}_{\mathrm{det}}\right]^{G^{\prime}}$. In particular, from the surjectivity $\mathbb{C}[Q] \rightarrow \mathbb{C}\left[\mathcal{X}_{\mathrm{det}}\right]$, we would get $\mathbb{C}^{p^{m} m}[Q]^{G^{\prime}} \neq 0$, hence $S^{p_{m} m}\left(Q^{*}\right)^{G^{\prime}} \neq 0$. This contradicts [Ho], Proposition 4.3 (a), if $p_{m} m<m^{2}$, i.e. if $m \geq 3$. Thus, $Z$ (and hence $\mathcal{X}_{\text {det }}$ ) is not normal.

3.9 Corollary. For any $m \geq 3$, and any nonzero homogeneous $f_{o} \in \mathbb{C}\left[\mathcal{X}_{\mathrm{det}}\right]{ }^{G^{\prime}}$ of positive degree, $\left\langle f_{o}\right\rangle$ is not a radical ideal of $\mathbb{C}\left[\mathcal{X}_{\mathrm{det}}\right]$. 
Proof. Let $\mathbb{C}\left(\mathcal{X}_{\mathrm{det}}\right)=\mathbb{C}\left(\mathcal{X}_{\mathrm{det}}^{o}\right)$ be the function field of $\mathcal{X}_{\mathrm{det}}$ (or $\left.\mathcal{X}_{\mathrm{det}}^{o}\right)$. As in the proof of the above theorem, $\mathcal{X}_{\text {det }}^{o}$ is affine and, of course, normal (in fact, smooth). Take a function $h \in \mathbb{C}\left(\mathcal{X}_{\mathrm{det}}\right)$ which is integral over $\mathbb{C}\left[\mathcal{X}_{\mathrm{det}}\right]$. Since $\mathcal{X}_{\mathrm{det}}^{o}$ is normal, $h \in \mathbb{C}\left[\mathcal{X}_{\mathrm{det}}^{o}\right]$. If $h \notin \mathbb{C}\left[\mathcal{X}_{\mathrm{det}}\right]$, we can write $h=h_{1} / f_{o}^{d_{o}}$ for some $d_{o}>0$ and $h_{1} \in \mathbb{C}\left[\mathcal{X}_{\text {det }}\right] \backslash\left\langle f_{o}\right\rangle$ (cf. Corollary 3.6 and [S], page 50). From this (and since $h$ is integral over $\mathbb{C}\left[\mathcal{X}_{\mathrm{det}}\right]$ ) we see that $h_{1}^{d} \in\left\langle f_{o}\right\rangle$ for some $d>0$. If $\left\langle f_{o}\right\rangle$ were a radical ideal, we would have $h_{1} \in\left\langle f_{o}\right\rangle$. This contradicts the choice of $h_{1}$. Hence $h \in \mathbb{C}\left[\mathcal{X}_{\text {det }}\right]$. Thus, $\mathcal{X}_{\text {det }}$ is normal, contradicting Theorem 3.8. This proves the corollary.

3.10 Remark. The saturation property fails for $\mathbb{C}\left[\mathcal{X}_{\mathrm{det}}\right]$ for $m=2$.

By [GW], page 296, as modules for $\operatorname{GL}(d)$ (for any $d \geq 1$ ),

$$
S\left(S^{2}\left(\mathbb{C}^{d}\right)\right) \simeq \underset{\mu \in 2 \sum_{i=1}^{d} \mathbb{Z}_{+} \omega_{i}}{\bigoplus} V(\mu),
$$

where $\omega_{i}:=\epsilon_{1}+\cdots+\epsilon_{i}$ is the $i$-th fundamental weight of $\operatorname{GL}(d)$. Observe that, for $m=2$, since $\mathcal{X}_{\text {det }}=Q$ (cf. Example 2.7), we have $\mathbb{C}\left[\mathcal{X}_{\mathrm{det}}\right]=S\left(S^{2}(E)\right.$ ). Thus, $V\left(2 \omega_{2}\right)$ appears in $S^{2}\left(S^{2}(E)\right)$, but $V\left(\omega_{2}\right)$ does not appear in $S^{1}\left(S^{2}(E)\right)$.

\section{Isotropy of permanent}

Consider the space $\mathfrak{v}$ of dimension $m$ as in Section 1. Fix a positive integer $n<m$. Choose a basis $\left\{e_{1}, \ldots, e_{m}\right\}$ of $\mathfrak{v}$ and consider the subspace $\mathfrak{v}_{1}$ of dimension $n$ spanned by $\left\{e_{m-n+1}, \ldots, e_{m}\right\}$. We identify End $\mathfrak{v}_{1}$ with the space of $n \times n$-matrices (under the basis $\left.\left\{e_{m-n+1}, \ldots, e_{m}\right\}\right)$. Then, the permanent of an $n \times n$-matrix gives rise to the function perm $\in S^{n}\left(\left(\text { End } \mathfrak{v}_{1}\right)^{*}\right)$. Consider the standard action of GL(End $\left.\mathfrak{v}_{1}\right)$ on $S^{n}\left(\left(\text { End } \mathfrak{v}_{1}\right)^{*}\right)$. In particular, GL(End $\left.\mathfrak{v}_{1}\right)$ acts on perm.

Recall the following from [MM] (cf. also [B]).

4.1 Proposition. For $n \geq 3$, the isotropy of perm under the action of the group $\mathrm{GL}\left(\right.$ End $\left.\mathfrak{v}_{1}\right)$ consists of the transformations

$$
\tau: X \mapsto \lambda X^{*} \mu,
$$

where $X^{*}$ is $X$ or $X^{t}$ and $\lambda, \mu$ belong to the subgroup $\hat{D}$ of $\operatorname{GL}\left(\mathfrak{v}_{1}\right)$ generated by the permutation matrices together with the diagonal matrices of determinant 1 .

Lemma 2.2 and its proof give the following.

4.2 Lemma. The determinant of the above map $\tau: X \mapsto \lambda X^{*} \mu$ is given by

$$
\begin{aligned}
\operatorname{det} \tau & =(-1)^{\frac{n(n-1)}{2}}(\operatorname{det} \lambda)^{n}(\operatorname{det} \mu)^{n} & & \text { if } X^{*}=X^{t}, \\
& =(\operatorname{det} \lambda)^{n}(\operatorname{det} \mu)^{n} & & \text { if } X^{*}=X .
\end{aligned}
$$


If particular, if $n=2 k$ for an odd integer $k$, then

$$
\begin{aligned}
& \operatorname{det} \tau=-1 \quad \text { if } X^{*}=X^{t} \text {, } \\
& =1 \text { if } X^{*}=X \text {. }
\end{aligned}
$$

4.3 Corollary. Let $n \geq 3$. Consider the homomorphism

$$
\gamma: \hat{D} \times \hat{D} \longrightarrow\left(\operatorname{GL}\left(\text { End } \mathfrak{v}_{1}\right)\right)_{\text {perm }}, \gamma(\lambda, \mu)(v \otimes f)=\lambda v \otimes\left(\mu^{-1}\right)^{*} f,
$$

for $v \otimes f \in \mathfrak{v}_{1} \otimes \mathfrak{v}_{1}^{*}=$ End $\mathfrak{v}_{1}$, where $\left(\mu^{-1}\right)^{*}$ denotes the map induced by $\mu^{-1}$ on the dual space $\mathfrak{v}_{1}^{*}$. Then, $\gamma$ induces an embedding of groups

$$
\bar{\gamma}:(\hat{D} \times \hat{D}) / \Theta_{n} \hookrightarrow\left(\mathrm{GL}\left(\text { End } \mathfrak{v}_{1}\right)\right)_{\text {perm }},
$$

where $\Theta_{n}$ acts on $\hat{D} \times \hat{D}$ via $z \cdot(\lambda, \mu)=(z \lambda, z \mu)$, for $z \in \Theta_{n}$.

Moreover, $\operatorname{Im} \bar{\gamma}$ contains the identity component of $\left(\mathrm{GL}\left(\text { End } \mathfrak{v}_{1}\right)\right)_{\text {perm }}$.

Further, if $n=2 k$ for an odd integer $k$, then $\bar{\gamma}$ is an isomorphism onto $\left(\operatorname{SL}\left(\text { End } \mathfrak{v}_{1}\right)\right)_{\text {perm }}$.

Since the isotropy $\operatorname{SL}\left(\text { End } \mathfrak{v}_{1}\right)_{\text {perm }}$ is not contained in any proper parabolic subgroup of SL(End $\left.\mathfrak{v}_{1}\right)$, Kempf's theorem [Ke], Corollary 5.1, gives the following result observed in [MS1], Theorem 4.7:

4.4 Proposition. For $n \geq 3$, SL(End $\left.\mathfrak{v}_{1}\right)$-orbit of perm inside $S^{n}\left(\left(\text { End }_{1}\right)^{*}\right)$ is closed.

Thus, an irreducible $\mathrm{SL}\left(\right.$ End $\left.\mathfrak{v}_{1}\right)$-module $M$ occurs in $\mathbb{C}\left[\overline{\mathrm{GL}\left(\text { End } \mathfrak{v}_{1}\right) \cdot \text { perm }}\right]$ if and only if $M^{\left(\mathrm{SL}\left(\mathrm{End} \mathfrak{v}_{1}\right)\right)_{\mathrm{perm}}} \neq 0$ (cf. the proof of Corollary 2.6).

By exactly the same proof as that of Theorem 3.8, we get the following:

4.5 Theorem. For $n \geq 3$, the subvariety $\overline{\mathrm{GL}\left(\text { End } \mathfrak{v}_{1}\right) \cdot \operatorname{perm}} \subset S^{n}\left(\left({\left.\left.\text { End } \mathfrak{v}_{1}\right)^{*}\right) \text { is not }}\right.\right.$ normal.

We prove the following lemma for its application in the next section.

4.6 Lemma. Let $C=\left(c_{i, j}\right) \in$ End $\mathfrak{v}_{1}$ be such that

$$
\operatorname{perm}(X+C)=\operatorname{perm}(X) \text { for all } X \in \text { End } \mathfrak{v}_{1} .
$$

Then, $C=0$.

Proof. Take $X=\left(x_{i, j}\right)$ with $x_{1,2}=\cdots=x_{1, n}=0$. Then,

$$
\operatorname{perm}(X)=\operatorname{perm}\left(\begin{array}{cccc}
x_{1,1} & 0 & \cdots & 0 \\
x_{2,1} & x_{2,2} & \cdots & x_{2, n} \\
\vdots & \vdots & & \vdots \\
x_{n, 1} & x_{n, 2} & \cdots & x_{n, n}
\end{array}\right)=x_{1,1} \text { perm } X^{(1,1)} \text {, }
$$


where

$$
X^{(1,1)}=\left(\begin{array}{ccc}
x_{2,2} & \cdots & x_{2, n} \\
\vdots & & \vdots \\
x_{n, 2} & \cdots & x_{n, n}
\end{array}\right) .
$$

By assumption, for any $X=\left(x_{i, j}\right)$ as above,

$$
\begin{aligned}
\operatorname{perm}(X)= & \operatorname{perm}(X+C) \\
= & \left(x_{1,1}+c_{1,1}\right) \operatorname{perm}\left(X^{(1,1)}+C^{(1,1)}\right)+c_{1,2} \operatorname{perm}\left(X^{(1,2)}+C^{(1,2)}\right) \\
& \quad+\cdots+c_{1, n} \operatorname{perm}\left(X^{(1, n)}+C^{(1, n)}\right) .
\end{aligned}
$$

Now, $x_{1,1}$ divides the left side by (7), hence it must also divide the right side of the above equation. Thus,

$$
\sum_{j=1}^{n} c_{1, j} \operatorname{perm}\left(X^{(1, j)}+C^{(1, j)}\right)=0
$$

and (by equations (7)-(9))

$$
\operatorname{perm}\left(X^{(1,1)}+C^{(1,1)}\right)=\operatorname{perm}\left(X^{(1,1)}\right) .
$$

By induction, this gives

$$
C^{(1,1)} \equiv 0
$$

By a similar argument,

$$
C^{(1, j)}=0 \text { for all } j .
$$

Substituting this in (9), we get

$$
\sum_{j=1}^{n} c_{1, j} \text { perm } X^{(1, j)}=0
$$

which gives $c_{1, j}=0$ for all $j$. Hence,

$$
C=0 \text {. }
$$

4.7 Remark. As pointed out by the referee, a similar proof shows that the above lemma is true for any $P \in S^{d}\left(\left(\mathbb{C}^{N}\right)^{*}\right)$ such that its zero set in $\mathbb{P}^{N-1}$ is not a cone.

\section{Functions on the orbit closure of $p$}

We take in this and the subsequent sections $3 \leq n<m$. 
Recall the definition of the subspace $\mathfrak{v}_{1} \subset \mathfrak{v}$ from Section 4. Let $\mathfrak{v}_{1}^{\perp}$ be the complementary subspace of $\mathfrak{v}$ with basis $\left\{e_{1}, \ldots, e_{m-n}\right\}$. Consider the padded permanent function $\mathrm{p} \in Q=S^{m}\left(E^{*}\right)$, defined by $\mathrm{p}(X)=x_{1,1}^{m-n} \operatorname{perm}\left(X^{o}\right), X^{o}$ being the component of $X$ in the right down $n \times n$ corner $\left(\begin{array}{lll}x_{1,1} & & * \\ & \ddots & \\ * & \underbrace{X^{o}}_{n}\end{array}\right)$, where any element of $E=$ End $\mathfrak{v}$ is represented by a $m \times m$-matrix $X=\left(x_{i, j}\right)_{1 \leq i, j, \leq m}$ in the basis $\left\{e_{i}\right\}$.

Let $S$ be the subspace of $E$ spanned by $e_{1,1}$ and $e_{i, j}, m-n+1 \leq i, j \leq$ $m$, and let $S^{\perp}$ be the complementary subspace spanned by the set $\left\{e_{i, j}\right\}_{1 \leq i, j, \leq m} \backslash$ $\left\{e_{1,1}, e_{i, j}\right\}_{m-n+1 \leq i, j \leq m}$ (where, as in Section $1, e_{i, j}:=e_{i} \otimes e_{j}^{*}$ ). Let $P$ be the maximal parabolic subgroup of $G=\operatorname{GL}(E)$ which keeps the subspace $S^{\perp}$ of $E$ stable. Let $U_{P}$ be the unipotent radical of $P$ and let $L_{P}$ be the Levi subgroup of $P$ defined by $L_{P}=\mathrm{GL}\left(S^{\perp}\right) \times \mathrm{GL}(S)$.

The following lemma is easy to verify.

5.1 Lemma. The subgroups $\operatorname{GL}\left(S^{\perp}\right)$ and $U_{P}$ act trivially on $\mathrm{p}$. Hence, $P \cdot \mathrm{p}=$ $\mathrm{GL}(S) \cdot \mathrm{p}$.

Since $G / P$ is a projective variety,

$$
X_{\mathrm{p}}:=G \cdot(\overline{P \cdot \mathrm{p}})=\overline{G \cdot \mathrm{p}} \subset Q .
$$

Thus, we have a proper surjective morphism

$$
\phi: G \times_{P}(\overline{P \cdot \mathrm{p}})=G \times_{P}(\overline{\mathrm{GL}(S) \cdot \mathrm{p}}) \rightarrow \mathcal{X}_{\mathrm{p}}, \quad[g, x] \mapsto g \cdot x,
$$

for $g \in G$ and $x \in \overline{P \cdot p}$. Consider the decomposition into irreducible components (for any $d \geq 0$ )

$$
\left.\mathbb{C}^{d}[\overline{\mathrm{GL}(S) \cdot \mathrm{p}}]=\bigoplus_{\lambda \in D(\mathrm{GL}(S))} n_{\lambda}(d) V_{\mathrm{GL}(S)}(\lambda)^{*} \quad \text { (for some } n_{\lambda}(d) \in \mathbb{Z}_{+}\right),
$$

where $\mathbb{C}^{d}[\overline{\mathrm{GL}(S) \cdot \mathrm{p}}]$ denotes the space of homogeneous degree $d$-functions with respect to the embedding $\overline{\operatorname{GL}(S) \cdot \mathrm{p}} \subset Q, D(\mathrm{GL}(S))$ denotes the set of dominant characters for the group $\operatorname{GL}(S)$ (with respect to its standard diagonal subgroup) consisting of $\lambda=\left(\lambda_{1} \geq \cdots \geq \lambda_{n^{2}+1}\right)$ with $\lambda_{i} \in \mathbb{Z}$, and $V_{\mathrm{GL}(S)}(\lambda)$ is the irreducible $\mathrm{GL}(S)$-module with highest weight $\lambda$.

For a certain generalization of the following theorem, see Proposition 6.3.2 of [BL].

5.2 Theorem. For any $\lambda \in D(\mathrm{GL}(S))$ and $d \geq 0$ such that $n_{\lambda}(d)>0$, we have $\lambda_{1} \leq 0$. 
Moreover, as G-modules,

$$
\mathbb{C}^{d}\left[\mathcal{X}_{\mathrm{p}}\right]=\bigoplus_{\lambda \in D(\mathrm{GL}(S))} n_{\lambda}(d) V_{G}(\hat{\lambda})^{*}
$$

where $\hat{\lambda}:=\left(0 \geq \cdots \geq 0 \geq \lambda_{1} \geq \cdots \geq \lambda_{n^{2}+1}\right) \in D(G)$ (with initial $m^{2}-n^{2}-1$ zeroes).

Further, the G-equivariant morphism $\phi$ induces an isomorphism of $G$-modules:

$$
\phi^{*}: \mathbb{C}\left[\mathcal{X}_{\mathrm{p}}\right] \rightarrow \mathbb{C}\left[G \times_{P}(\overline{P \cdot \mathrm{p}})\right] .
$$

Proof. Observe that, by Lemma 5.1, $\mathbb{C}^{d}[\overline{\mathrm{GL}}(S) \cdot \mathrm{p}]$ is a $P$-module quotient of $\mathbb{C}^{d}[\overline{G \cdot \mathrm{p}}]$ with $U_{P}$ and $\mathrm{GL}\left(S^{\perp}\right)$ acting trivially on $\mathbb{C}^{d}[\overline{\mathrm{GL}}(S) \cdot \mathrm{p}]$. Thus, as $P$ modules,

$$
\mathbb{C}^{d}[\overline{\mathrm{GL}(S) \cdot \mathrm{p}}]^{*} \simeq \bigoplus_{\lambda \in D(\mathrm{GL}(S))} n_{\lambda}(d) V_{\mathrm{GL}(S)}(\lambda) \hookrightarrow \mathbb{C}^{d}\left[\mathcal{X}_{\mathrm{p}}\right]^{*} .
$$

Take a nonzero $B_{\mathrm{GL}(S)}$-eigenvector of weight $\lambda$ in $\mathbb{C}^{d}[\overline{\mathrm{GL}(S) \cdot \mathrm{p}}]^{*}$, where $B_{\mathrm{GL}(S)}$ is the standard Borel subgroup of $\mathrm{GL}(S)$ consisting of upper triangular matrices. Then, its image in $\mathbb{C}^{d}\left[\mathcal{X}_{\mathrm{p}}\right]^{*}$ is a $B$-eigenvector of weight $\hat{\lambda}$, where $B$ is the standard Borel subgroup of $G$. In particular, for any $\lambda \in D(\operatorname{GL}(S))$ such that $n_{\lambda}(d)>0, \hat{\lambda} \in D(G)$ (since $\mathbb{C}^{d}\left[X_{\mathrm{p}}\right]^{*}$ is a $G$-module). Hence, $\lambda_{1} \leq 0$ and $\bigoplus_{\lambda \in D(\operatorname{GL}(S))} n_{\lambda}(d) V_{G}(\hat{\lambda}) \subset$ $\mathbb{C}^{d}\left[\mathcal{X}_{\mathrm{p}}\right]^{*}$. Dualizing, we get the $G$-module surjection:

$$
\mathbb{C}^{d}\left[\mathcal{X}_{\mathrm{p}}\right] \rightarrow \bigoplus_{\lambda \in D(\mathrm{GL}(S))} n_{\lambda}(d) V_{G}(\hat{\lambda})^{*} .
$$

From the surjection $\phi$, we obtain the $G$-module injective map:

$$
\begin{aligned}
\phi^{*}: \mathbb{C}^{d}\left[\mathcal{X}_{\mathrm{p}}\right] \hookrightarrow & H^{0}\left(G / P, \mathbb{C}^{d}[\overline{\mathrm{GL}(S) \cdot \mathrm{p}}]\right) \\
& =\bigoplus_{\lambda \in D(\mathrm{GL}(S))} n_{\lambda}(d) H^{0}\left(G / P, V_{\mathrm{GL}(S)}(\lambda)^{*}\right),
\end{aligned}
$$

where $U_{P}$ and $\mathrm{GL}\left(S^{\perp}\right)$ act trivially on $V_{\mathrm{GL}(S)}(\lambda)^{*}$

$$
\simeq \bigoplus_{\lambda \in D(G L(S))} n_{\lambda}(d) V_{G}(\hat{\lambda})^{*},
$$

where the last isomorphism follows from [Ku1], Lemma 8. Combining the injection $\phi^{*}$ with (11), we get that $\phi^{*}$ is an isomorphism, proving the theorem.

5.3 Proposition. The isotropy of $\mathrm{p}$ under the group $P$ is the same as that under the group $G$. 
Proof. First of all $G / P=W_{P}^{\prime} U_{P}^{-} P / P$, where $U_{P}^{-}$is the opposite of the unipotent radical $U_{P}$ of $P$ and $W_{P}^{\prime}$ is the set of all the smallest coset representatives of $W / W_{P}$, $W$ (resp. $W_{P}$ ) being the Weyl group of $G$ (resp. $P$ ). (This follows since the right side is an open subset of $G / P$ which is $T$-stable and contains all the $T$-fixed points of $G / P$.)

Take $w \in W_{P}^{\prime}, u \in U_{P}^{-}, r \in \operatorname{GL}(S)$ such that $w u r \cdot \mathrm{p}=\mathrm{p}$. Then,

$$
\mathrm{p}\left(r^{-1} u^{-1} w^{-1} X\right)=\mathrm{p}(X) \text { for any } X=X_{1}+X_{2} \in E=S^{\perp} \oplus S .
$$

In particular, for $X=w X_{2}$, we get

$$
\mathrm{p}\left(r^{-1} u^{-1} X_{2}\right)=\mathrm{p}\left(w X_{2}\right) .
$$

We have $u^{-1} X_{2}=X_{2}$, thus

$$
\mathrm{p}\left(r^{-1} u^{-1} X_{2}\right)=\mathrm{p}\left(r^{-1} X_{2}\right) .
$$

Now, well-order a basis of $S$ as $v_{1}, v_{2}, \ldots, v_{d}\left(d=n^{2}+1\right)$ and also a basis $v_{d+1}, \ldots, v_{m^{2}}$ of $S^{\perp}$. Then, $w$ can be represented as the permutation $i \mapsto n_{i}$ with

$$
n_{1}<\cdots<n_{d}, n_{d+1}<\cdots<n_{m^{2}} .
$$

For $X_{2}=\sum_{i=1}^{d} z_{i} v_{i} \in S$,

$$
\mathrm{p}\left(w X_{2}\right)=\mathrm{p}\left(\sum_{i=1}^{d} z_{i} v_{n_{i}}\right)=\mathrm{p}\left(\sum_{i \leq i_{o}} z_{i} v_{n_{i}}\right),
$$

where $1 \leq i_{o} \leq d$ is the maximum integer such that $n_{i_{o}} \leq d$. In particular, $\mathrm{p}\left(w X_{2}\right)$ only depends upon the variables $z_{1}, \ldots, z_{i_{o}}$. Thus, by the identities (13)-(15),

$$
\mathrm{p}\left(r^{-1} \sum_{i=1}^{d} z_{i} v_{i}\right)=\mathrm{p}\left(\sum_{i \leq i_{o}} z_{i} v_{n_{i}}\right) \quad \text { for any } z_{i} \in \mathbb{C},
$$

which gives

$$
\mathrm{p}\left(r^{-1} \sum_{i=1}^{d} z_{i} v_{i}\right)=\mathrm{p}\left(r^{-1}\left(\sum_{i=1}^{d} z_{i} v_{i}+\sum_{d \geq j>i_{o}} b_{j} v_{j}\right)\right) \quad \text { for any } b_{j} \in \mathbb{C} .
$$

Thus,

$$
\mathrm{p}\left(\sum_{i=1}^{d} z_{i} v_{i}\right)=\mathrm{p}\left(\sum_{i=1}^{d} z_{i} v_{i}+r^{-1} \sum_{d \geq j>i_{o}} b_{j} v_{j}\right) .
$$

Applying Lemma 4.6, it is easy to see that $\sum_{d \geq j>i_{o}} b_{j} v_{j}=0$ (for any $b_{j} \in \mathbb{C}$ ). Thus, $i_{o}=d$, i.e., $w=1$. 
Taking $X=X_{2} \in S$ in (12), we get (since $\left.w=1\right) \mathrm{p}\left(r^{-1} X_{2}\right)=\mathrm{p}\left(X_{2}\right)$, which is equivalent to $\mathrm{p}\left(r^{-1} X\right)=\mathrm{p}(X)$ for all $X \in E$. Thus, $r$ is in the isotropy of $\mathrm{p}$ and hence $u$ is in the isotropy of p, i.e., $\mathrm{p}\left(u^{-1} X\right)=\mathrm{p}(X)$ for all $X=X_{1}+X_{2} \in E$. This gives $\mathrm{p}\left(X_{1}+X_{2}+Y_{2}\right)=\mathrm{p}\left(X_{1}+X_{2}\right)$, where $Y_{2}:=u^{-1} X_{1}-X_{1} \in S$. Hence, $\mathrm{p}\left(X_{2}+Y_{2}\right)=\mathrm{p}\left(X_{2}\right)$ for all $X_{2} \in S$ and any $Y_{2}$ of the form $u^{-1} X_{1}-X_{1}$, for some $X_{1} \in S^{\perp}$. Applying Lemma 4.6 again, we see that $Y_{2}=0$, hence $u_{\left.\right|_{S} \perp}=$ Id. Thus, $u=1$. This proves the proposition since $U_{P}$ and $\operatorname{GL}\left(S^{\perp}\right)$ stabilize $\mathrm{p}$.

5.4 Corollary. The restriction $\phi_{o}$ of the map $\phi$ to $G \times_{P}(P \cdot p)$ is a biregular isomorphism onto $G \cdot \mathrm{p}$.

Moreover, $\phi^{-1}(G \cdot \mathrm{p})=G \times_{P}(P \cdot \mathrm{p})$.

Proof. Of course, $\phi_{o}$ is surjective. We next claim that $\phi_{o}$ is injective. Take $\phi_{o}[g, \mathrm{p}]=$ $\phi_{o}\left[g_{1}, \mathrm{p}\right]$, i.e., $g \cdot \mathrm{p}=g_{1} \cdot \mathrm{p}$, which is equivalent to $\left(g_{1}^{-1} g\right) \cdot \mathrm{p}=\mathrm{p}$, i.e., $g_{1}^{-1} g \in$ $G_{\mathrm{p}}=P_{\mathrm{p}}$, by Proposition 5.3. Thus, $g_{1}^{-1} g=\tilde{r}$ for some $\tilde{r} \in P_{\mathrm{p}} \subset P$. Hence, $[g, \mathrm{p}]=\left[g_{1}, \mathrm{p}\right]$, proving that $\phi_{o}$ is bijective. Since $G \times \times_{P}(P \cdot \mathrm{p})$ and $G \cdot \mathrm{p}$ are both smooth, $\phi_{o}$ is an isomorphism (cf. [Ku2], Theorem A.11).

To prove that $\phi^{-1}(G \cdot \mathrm{p})=G \times{ }_{P}(P \cdot \mathrm{p})$, take $[g, y] \in G \times \times_{P}(\overline{P \cdot \mathrm{p}})$ such that $\phi[g, y] \in G \cdot \mathrm{p}$, i.e., $g \cdot y=h \cdot \mathrm{p}$ for some $h \in G$. This gives $y \in G \cdot \mathrm{p} \cap \overline{P \cdot \mathrm{p}}$. But, $P \cdot \mathrm{p}$ is closed in $G \cdot \mathrm{p}$ by the first part of the corollary and hence $y \in P \cdot \mathrm{p}$, establishing the claim.

Let $S_{1}$ be the subspace of $S$ spanned by $e_{i, j}, m-n+1 \leq i, j \leq m$. Consider the maximal parabolic subgroup $R$ of $\mathrm{GL}(S)=$ Aut $S$, consisting of those $g \in$ Aut $S$ which stabilize the line $\mathbb{C} e_{1,1}$. Then, $L_{R}:=\operatorname{Aut}\left(\mathbb{C} e_{1,1}\right) \times \operatorname{Aut} S_{1}$ is a Levi subgroup of $R$. Let $U_{R}$ be the unipotent radical of $R$ and $U_{R}^{-}$the opposite unipotent radical.

5.5 Proposition. The isotropy of $\mathrm{p}$ under the group $\mathrm{GL}(S)$ is the same as the isotropy of the Levi subgroup $L_{R}$.

Proof. In the proof, we let $i, j$ run over $m-n+1 \leq i, j \leq m$. Any element $u \in U_{R}$ is given by $u e_{1,1}=e_{1,1}, u e_{i, j}=e_{i, j}+a_{i, j} e_{1,1}$, for some $a_{i, j} \in \mathbb{C}$. Similarly, $U_{R}^{-}$ consists of $u^{-}$such that $u^{-} e_{i, j}=e_{i, j}$ and $u^{-} e_{1,1}=e_{1,1}+\sum c_{i, j} e_{i, j}$. Any element of GL(S) can be written as $w u^{-} u g$ (for some $g \in L_{R}, u \in U_{R}, u^{-} \in U_{R}^{-}$and $w$ either the identity element or a 2-cycle $((1,1),(i, j)))$. Take any $X=x_{1,1} e_{1,1}+$ $\sum x_{i, j} e_{i, j} \in S$. By $X_{S_{1}}$ we mean $\sum x_{i, j} e_{i, j}$ and by $(X)_{1,1}$ we mean $x_{1,1}$.

$$
\begin{aligned}
\left(\left(w u^{-} u g\right)^{-1} \cdot p\right)(X) & =p\left(w u^{-} u g X\right) \\
& =\left(\left(w u^{-} u g X\right)_{1,1}\right)^{m-n} \operatorname{perm}\left(\left(w u^{-} u g X\right)_{S_{1}}\right) .
\end{aligned}
$$

So, if $\left(w u^{-} u g\right)^{-1} \in(\operatorname{GL}(S))_{p}$, then

$$
\left(\left(w u^{-} u g\right)^{-1} \cdot \mathrm{p}\right)(X)=\mathrm{p}(X)=x_{1,1}^{m-n} \operatorname{perm}\left(X_{S_{1}}\right) \quad \text { for all } X \in S .
$$


Since no linear form divides perm, we get

$$
\alpha x_{1,1}=\left(w u^{-} u g X\right)_{1,1} \quad \text { for some constant } \alpha \neq 0 \in \mathbb{C},
$$

and

$$
\begin{aligned}
\beta \operatorname{perm}\left(X_{S_{1}}\right) & =\operatorname{perm}\left(\left(w u^{-} u g X\right)_{S_{1}}\right) \quad \text { for some constant } \beta \neq 0 \in \mathbb{C} \\
& =\operatorname{perm}\left(\left(w u^{-} u g\left(X_{S_{1}}\right)+x_{1,1} w u^{-} u g e_{1,1}\right)_{S_{1}}\right) .
\end{aligned}
$$

Since the left hand side of (17) is independent of $x_{1,1}$, we get

$$
\operatorname{perm}\left(\left(w u^{-} u g X\right)_{S_{1}}\right)=\operatorname{perm}\left(\left(w u^{-} u g X\right)_{S_{1}}+\left(\alpha_{1,1} w u^{-} u g e_{1,1}\right)_{S_{1}}\right),
$$

for all $X \in S$ and $\alpha_{1,1} \in \mathbb{C}$.

Since $w u^{-} u g \in$ Aut $S$, as $X$ varies over $S,\left(w u^{-} u g X\right)_{S_{1}}$ varies over all of $S_{1}$. Thus, by Lemma 4.6,

$$
\left(w u^{-} u g e_{1,1}\right)_{S_{1}}=0 .
$$

Now,

$$
\begin{aligned}
u^{-} u g e_{1,1} & =u^{-}\left(\lambda e_{1,1}\right) \quad \text { for some } \lambda \neq 0 \\
& =\lambda\left(e_{1,1}+\sum c_{i, j} e_{i, j}\right) .
\end{aligned}
$$

Thus, if $w$ is the 2-cycle $\left((1,1),\left(i_{o}, j_{o}\right)\right)$ for some $m-n+1 \leq i_{o}, j_{o} \leq m$, then

$$
w u^{-} u g e_{1,1}=\lambda\left(e_{i_{o}, j_{o}}+\sum_{(i, j) \neq\left(i_{o}, j_{o}\right)} c_{i, j} e_{i, j}+c_{i_{o}, j_{o}} e_{1,1}\right) \text {. }
$$

In particular, $\left(w u^{-} u g e_{1,1}\right) s_{1} \neq 0$, a contradiction to the identity (18). Thus, $w=1$. By the equations (18)- (19), we get

$$
c_{i, j}=0 \quad \text { for all } i, j \text {. }
$$

Thus, $u^{-}=1$.

By equation (16), we get

$$
\alpha x_{1,1}=\left(w u^{-} u g X\right)_{1,1}=(u g X)_{1,1}=\left(u g\left(X_{S_{1}}+x_{1,1} e_{1,1}\right)\right)_{1,1} .
$$

In particular, $\left(u g X_{S_{1}}\right)_{1,1}=0$. Since $g$ maps $S_{1}$ onto $S_{1}$, we get

$$
\left(u e_{i, j}\right)_{1,1}=0 \text { for all } m-n+1 \leq i, j \leq m \text {. }
$$

Hence, $a_{i, j}=0$. Thus, $u=1$ as well. This proves the proposition.

5.6 Corollary. Let $3 \leq n<m$. Then, each irreducible component of

$$
\overline{\mathrm{GL}(S) \cdot \mathrm{p}} \backslash(\mathrm{GL}(S) \cdot \mathrm{p})
$$

is of codimension 1 in $\overline{\mathrm{GL}(S) \cdot \mathrm{p}}$. 
Proof. By the last proposition, the isotropy of $\mathrm{p}$ inside $\mathrm{GL}(S)$ is the same as that of the isotropy of $\mathrm{p}$ inside $L_{R}$. For any $\lambda \in \mathbb{C}^{*}$, take $\tau_{\lambda} \in \operatorname{Aut}\left(\mathbb{C} e_{1,1}\right)$ defined by $e_{1,1} \mapsto \lambda e_{1,1}$. Then, for any $g \in$ Aut $S_{1}$ and $X=x_{1,1} e_{1,1}+X_{1}$ with $X_{1} \in S_{1}$, we have

$$
\begin{aligned}
\left(\left(\tau_{\lambda}, g\right) \cdot p\right)(X) & =p\left(\lambda^{-1} x_{1,1} e_{1,1}+g^{-1} X_{1}\right) \\
& =\left(\lambda^{-1} x_{1,1}\right)^{m-n} \operatorname{perm}\left(g^{-1} X_{1}\right) .
\end{aligned}
$$

Thus, $\left(\tau_{\lambda}, g\right) \in\left(L_{R}\right)_{\mathrm{p}}$ if and only if $\left(\lambda^{\frac{1}{n}}\right)^{m-n} g \in\left(\text { Aut } S_{1}\right)_{\text {perm }}$ for some $n$-th root $\lambda^{\frac{1}{n}}$ of $\lambda$. Considering the projection to the first factor $\left(L_{R}\right)_{\mathrm{p}} \rightarrow \operatorname{Aut}\left(\mathbb{C} e_{1,1}\right)=\mathbb{C}^{*}$ and using Corollary 4.3 , it is easy to see that $\left(L_{R}\right)_{\mathrm{p}}=(\operatorname{GL}(S))_{\mathrm{p}}$ is reductive. Thus, $\mathrm{GL}(S) \cdot \mathrm{p}$ is an affine variety. Of course, $\overline{\operatorname{GL}(S) \cdot \mathrm{p}}$ is an affine variety. Moreover, $0 \in(\overline{\operatorname{GL}(S) \cdot p)} \backslash(\operatorname{GL}(S) \cdot p)$ by (20). Thus, $(\overline{\operatorname{GL}(S) \cdot p)} \backslash(\operatorname{GL}(S) \cdot p)$ is nonempty and each of its irreducible components is of codimension 1 in $\overline{\mathrm{GL}(S) \cdot p}$ by the following lemma.

We recall the following well-known result from algebraic geometry. For the lack of reference, we include a proof.

5.7 Lemma. Let $X$ be an irreducible affine variety and let $X^{\circ} \subset X$ be an open normal affine subvariety. Then, each irreducible component of $X \backslash X^{o}$ is of codimension 1 in $X$.

Proof. Let $\pi: \tilde{X} \rightarrow X$ be the normalization of $X$. Then, $X^{o}$ being normal and open subvariety of $X, \pi: \pi^{-1}\left(X^{o}\right) \rightarrow X^{o}$ is an isomorphism. We identify $\pi^{-1}\left(X^{o}\right)$ with $X^{o}$ under $\pi$. Decompose $\tilde{X} \backslash X^{o}=C_{1} \cup C_{2}$, where $C_{1}$ (resp. $C_{2}$ ) is the union of codimension 1 (resp. $\geq 2$ ) irreducible components of $\tilde{X} \backslash X^{o}$. Then, by Hartog's theorem, the inclusion $i: X^{o} \subset \tilde{X} \backslash C_{1}$ induces an isomorphism $i^{*}: \mathbb{C}\left[\widetilde{X} \backslash C_{1}\right] \simeq$ $\mathbb{C}\left[X^{o}\right]$ of the rings of regular functions. Let $f$ be the inverse of $i^{*}$. Then, $X^{o}$ being affine, there exists a morphism $j: \tilde{X} \backslash C_{1} \rightarrow X^{o}$ such that the induced map $j^{*}=f$ and $j_{\mid X^{o}}=\mathrm{Id}(\mathrm{cf}$. [H], Proposition 3.5, Chapter I). Since the composite morphism $i \circ j: \tilde{X} \backslash C_{1} \rightarrow \tilde{X} \backslash C_{1}$ restricts to the identity map on $X^{o}$ and $X^{o}$ is dense in $\tilde{X} \backslash C_{1}, i \circ j=$ Id. In particular, $i$ is surjective, i.e., $X^{o}=\tilde{X} \backslash C_{1}$. Thus,

$$
X \backslash X^{o}=\pi\left(\tilde{X} \backslash X^{o}\right)=\pi\left(C_{1}\right) .
$$

But, since $\pi$ is a finite morphism, $\pi\left(C_{1}\right)$ is closed in $X$ and, moreover, all the irreducible components of $\pi\left(C_{1}\right)$ are of codimension 1 in $X$.

As another corollary of Proposition 5.5 (together with Corollary 4.3, Lemma 5.1, Proposition 5.3 and identity (20)), we get the following well-known result.

5.8 Corollary. For $3 \leq n<m$, $\operatorname{dim} X_{p}=m^{2}\left(n^{2}+1\right)-2 n+1$. 


\section{A partial desingularization of $\overline{\operatorname{GL}(S) \cdot p}$}

By virtue of the results in the last section (specifically Theorem 5.2), study of the $G$-module $\mathbb{C}\left[\mathcal{X}_{\mathrm{p}}\right]$ reduces to that of the $\operatorname{GL}(S)$-module $\mathbb{C}[\overline{\operatorname{GL}(S) \cdot \mathrm{p}}]$.

6.1 Definition. Define the morphism

$$
\beta: \operatorname{GL}(S) \times_{R}(\overline{R \cdot \mathrm{p}}) \rightarrow \overline{\mathrm{GL}(S) \cdot \mathrm{p}}, \quad[g, f] \mapsto g \cdot f,
$$

for $g \in \mathrm{GL}(S), f \in \overline{R \cdot \mathrm{p}}$, where the closure $\overline{R \cdot \mathrm{p}}$ is taken inside $S^{m}\left(E^{*}\right)$.

Since $\operatorname{GL}(S) / R$ is a projective variety, $\beta$ is a proper and surjective morphism.

6.2 Lemma. The restriction $\beta_{o}$ of $\beta$ to $\operatorname{GL}(S) \times_{R}(R \cdot p)$ is a biregular isomorphism onto $\mathrm{GL}(S) \cdot \mathrm{p}$. Moreover, the inverse image $\beta^{-1}(\mathrm{GL}(S) \cdot p)$ equals $\mathrm{GL}(S) \times_{R}(R \cdot p)$.

Proof. By Proposition 5.5, the isotropy of $\mathrm{p}$ inside $\mathrm{GL}(S)$ is the same as that in $R$. From this the injectivity of $\beta_{o}$ follows easily. Since $\beta_{o}$ is a bijective morphism between smooth varieties, it is a biregular isomorphism.

Take $[g, f] \in \beta^{-1}(\operatorname{GL}(S) \cdot \mathrm{p})$. Then, $f \in(\mathrm{GL}(S) \cdot \mathrm{p}) \cap \overline{R \cdot \mathrm{p}}$. But, since $\beta_{o}$ is an isomorphism, $R \cdot p$ is closed in $\operatorname{GL}(S) \cdot p$. Thus, $(\operatorname{GL}(S) \cdot p) \cap \overline{R \cdot p}=R \cdot p$. This proves the second part of the lemma.

As in Section 4, consider perm $\in S^{n}\left(S_{1}^{*}\right)$, where $S_{1}$ is viewed as End $\mathfrak{v}_{1}$ and $\mathfrak{v}_{1}$ is equipped with the basis $\left\{e_{m-n+1}, \ldots, e_{m}\right\}$. Moreover, the decomposition $E=$ $S^{\perp} \oplus \mathbb{C} e_{1,1} \oplus S_{1}$ gives rise to the projection $E \rightarrow S_{1}$ and, in turn, an embedding $S^{n}\left(S_{1}^{*}\right) \hookrightarrow S^{n}\left(E^{*}\right)$. Thus, we can think of perm $\in S^{n}\left(E^{*}\right)$. Let

$$
X_{\text {perm }}^{o}:=\left(\text { Aut } S_{1}\right) \cdot \text { perm } \subset S^{n}\left(E^{*}\right),
$$

where Aut $S_{1}$ is to be thought of as the subgroup of $G$ by extending any automorphism of $S_{1}$ to that of $E$ by defining it to be the identity map on $S^{\perp} \oplus \mathbb{C} e_{1,1}$. Let $\mathcal{X}_{\text {perm }}$ be the closure of $\mathcal{X}_{\text {perm }}^{o}$ in $S^{n}\left(E^{*}\right)$.

Consider the standard (dual) action of $\operatorname{GL}(S)=$ Aut $S$ on $S^{*}$. In particular, we get an action of $R$ on $S^{*}$. Also, it is easy to see that $U_{R}$ and $\operatorname{Aut}\left(\mathbb{C} e_{11}\right)$ act trivially on $\chi_{\text {perm }}^{o}$ (and hence on $\mathcal{X}_{\text {perm }}$ ) under the standard action of $G$ on $S^{n}\left(E^{*}\right)$. In particular, $\chi_{\text {perm }}$ is a $R$-stable closed subset of $S^{n}\left(E^{*}\right)$ (under the standard action of $R$ ).

Consider the morphism

$$
\bar{\alpha}: S^{*} \times X_{\text {perm }} \rightarrow Q, \quad(\lambda, f) \mapsto \bar{\lambda}^{m-n} f,
$$

for $\lambda \in S^{*}$ and $f \in \mathcal{X}_{\text {perm }}$, where $\bar{\lambda} \in E^{*}$ is the image of $\lambda$ under the inclusion $S^{*} \hookrightarrow E^{*}$ induced from the projection $E \rightarrow S$. Then, $\bar{\alpha}$ is $R$-equivariant under the diagonal action of $R$ on $S^{*} \times \mathcal{X}_{\text {perm. }}$. Define an action of $\mathbb{C}^{*}$ on $S^{*} \times \mathcal{X}_{\text {perm }}$ via

$$
z(\lambda, f)=\left(z \lambda, z^{n-m} f\right) .
$$


This action commutes with the action of $R$. Then, $\bar{\alpha}$ clearly factors through the $\mathbb{C}^{*}$-orbits, and hence we get an $R$-equivariant morphism

$$
\alpha:\left(S^{*} \times \mathcal{X}_{\text {perm }}\right) / / \mathbb{C}^{*} \rightarrow Q .
$$

6.3 Proposition. The above morphism $\alpha$ is a finite morphism with image precisely equal to $\overline{R \cdot \mathrm{p}}$.

Moreover, $\alpha^{-1}(R \cdot \mathrm{p})=\left(\left(S^{*} \backslash S_{1}^{*}\right) \times \mathcal{X}_{\text {perm }}^{o}\right) / / \mathbb{C}^{*}$ and the map $\alpha_{o}$ obtained from the restriction of $\alpha$ to $\left(\left(S^{*} \backslash S_{1}^{*}\right) \times \mathcal{X}_{\text {perm }}^{o}\right) / / \mathbb{C}^{*}$ is a biregular isomorphism

$$
\alpha_{o}:\left(\left(S^{*} \backslash S_{1}^{*}\right) \times \mathcal{X}_{\text {perm }}^{o}\right) / / \mathbb{C}^{*} \stackrel{\sim}{\longrightarrow} R \cdot \mathrm{p},
$$

where $S_{1}^{*}$ is thought of as a subspace of $S^{*}$ via the projection $S=\mathbb{C} e_{1,1} \oplus S_{1} \rightarrow S_{1}$.

In particular, $\alpha$ is a proper and birational morphism onto $\overline{R \cdot \mathrm{p}}$.

Proof. Consider the $\mathbb{C}^{*}$-equivariant closed embedding

$$
S^{*} \times \mathcal{X}_{\text {perm }} \hookrightarrow E^{*} \times S^{n}\left(E^{*}\right),
$$

where $\mathbb{C}^{*}$ acts on the right side by the same formula as (21). This gives rise to the closed embedding

$$
\iota:\left(S^{*} \times \mathcal{X}_{\text {perm }}\right) / / \mathbb{C}^{*} \hookrightarrow\left(E^{*} \times S^{n}\left(E^{*}\right)\right) / / \mathbb{C}^{*} .
$$

We next claim that the morphism

$$
\psi:\left(E^{*} \times S^{n}\left(E^{*}\right)\right) / / \mathbb{C}^{*} \rightarrow Q=S^{m}\left(E^{*}\right),
$$

induced from the map $(\bar{\lambda}, f) \mapsto \bar{\lambda}^{m-n} f$, for $\bar{\lambda} \in E^{*}$ and $f \in S^{n}\left(E^{*}\right)$, is a finite morphism. Define a new $\mathbb{C}^{*}$ action on $E^{*} \times S^{n}\left(E^{*}\right)$ by

$$
t \odot(\bar{\lambda}, f)=(t \bar{\lambda}, t f) \quad \text { for } t \in \mathbb{C}^{*} .
$$

This $\mathbb{C}^{*}$-action commutes with the $\mathbb{C}^{*}$-action given by (21). Thus, we get a $\mathbb{C}^{*}$ action (still denoted by $\odot$ ) on $\left(E^{*} \times S^{n}\left(E^{*}\right)\right) / / \mathbb{C}^{*}$. Also, define a new $\mathbb{C}^{*}$-action on $S^{m}\left(E^{*}\right)$ by

$$
t \odot f=t^{m-n+1} f \quad \text { for } t \in \mathbb{C}^{*} \text { and } f \in S^{m}\left(E^{*}\right) .
$$

Then, $\psi$ is $\mathbb{C}^{*}$-equivariant. Moreover, $\psi^{-1}(0)=\left(0 \times S^{n}\left(E^{*}\right) \cup E^{*} \times 0\right) / / \mathbb{C}^{*}=$ $\{0\}$. Thus, by Lemma 3.2 (applied to the map $\psi$ considered as a map: $\left(E^{*} \times\right.$ $\left.\left.S^{n}\left(E^{*}\right)\right) / / \mathbb{C}^{*} \rightarrow \overline{\operatorname{Im} \psi}\right), \psi$ is a finite morphism.

Since $\alpha=\psi \circ \iota$, we get that $\alpha$ is a finite morphism.

We next calculate $\alpha^{-1}(\mathrm{p})$. Let $[\lambda, f] \in \alpha^{-1}(\mathrm{p})$, where $[\lambda, f]$ denotes the image of $(\lambda, f)$ in $\left(S^{*} \times X_{\text {perm }}\right) / / \mathbb{C}^{*}$. Then,

$$
\bar{\lambda}^{m-n} f=\mathrm{p}=\bar{\lambda}_{o}^{m-n} \text { perm },
$$


where $\lambda_{o} \in S^{*}$ is defined by $\lambda_{o}\left(z e_{1,1}+X_{1}\right)=z$ for any $z \in \mathbb{C}$ and $X_{1} \in S_{1}$.

Since $\bar{\lambda}$ does not divide perm, from (22) we get

$$
\lambda=a \lambda_{o} \text { and } f=a^{n-m} \text { perm for some } a \in \mathbb{C}^{*},
$$

which gives

$$
[\lambda, f]=\left[\lambda_{o}, \text { perm }\right] .
$$

Thus, $\alpha^{-1}(\mathrm{p})$ is a singleton and hence so is $\alpha^{-1}(r \cdot \mathrm{p})$ for any $r \in R$ (by the $R$ equivariance of $\alpha$ ). In particular,

$$
\begin{array}{rlr}
\alpha^{-1}(R \cdot \mathrm{p}) & =R \cdot\left[\lambda_{o}, \text { perm }\right] \\
& =\left(\operatorname{Aut}\left(\mathbb{C} e_{1,1}\right) U_{R} \operatorname{Aut}\left(S_{1}\right)\right) \cdot\left[\lambda_{o}, \text { perm }\right] \\
& =\left(\operatorname{Aut}\left(\mathbb{C} e_{1,1}\right) U_{R}\right) \cdot\left[\lambda_{o}, X_{\text {perm }}^{o}\right], & \text { since } \operatorname{Aut}\left(S_{1}\right) \cdot \lambda_{o}=\lambda_{o} \\
& =\left[\left(\operatorname{Aut}\left(\mathbb{C} e_{1,1}\right) U_{R}\right) \cdot \lambda_{o}, X_{\text {perm }}^{o}\right], & \text { since } \operatorname{Aut}\left(\mathbb{C} e_{1,1}\right) \text { and } \\
& & \\
& =\left[S_{R} \text { act trivially on } \mathcal{X}_{\text {perm }}^{o} \backslash S_{1}^{*}, \mathcal{X}_{\text {perm }}^{o}\right] & \\
& =\left(\left(S^{*} \backslash S_{1}^{*}\right) \times X_{\text {perm }}^{o}\right) / / \mathbb{C}^{*} .
\end{array}
$$

Observe that all the $\mathbb{C}^{*}$-orbits in $\left(S^{*} \backslash S_{1}^{*}\right) \times \mathcal{X}_{\text {perm }}^{o}$ are closed in $S^{*} \times \mathcal{X}_{\text {perm }}$ and hence $\left(\left(S^{*} \backslash S_{1}^{*}\right) \times \mathcal{X}_{\text {perm }}^{o}\right) / / \mathbb{C}^{*}=\left(\left(S^{*} \backslash S_{1}^{*}\right) \times \mathcal{X}_{\text {perm }}^{o}\right) / \mathbb{C}^{*}$ can be thought of as an open subset of $\left(S^{*} \times \mathcal{X}_{\text {perm }}\right) / / \mathbb{C}^{*}$. This proves that $\alpha_{o}$ is a bijective morphism between smooth irreducible varieties and hence it is a biregular isomorphism (cf. [Ku2], Theorem A.11).

Finally, since $\alpha$ is a finite morphism (in particular, a proper morphism), $\operatorname{Im} \alpha$ is

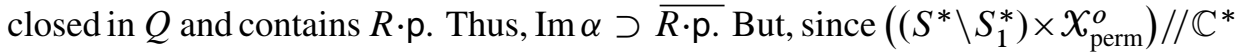
is dense in $S^{*} \times \mathcal{X}_{\text {perm }} / / \mathbb{C}^{*}$, we get $\operatorname{Im} \alpha \subset \overline{R \cdot \mathrm{p}}$ and hence $\operatorname{Im} \alpha=\overline{R \cdot \mathrm{p}}$.

This completes the proof of the proposition.

6.4 Remark. Even though we do not need, the above map $\alpha$ is a bijection onto its image.

Combining Lemma 6.2 with Proposition 6.3, we get the following:

6.5 Corollary. We have

$$
\begin{aligned}
& \mathbb{C}[\overline{\mathrm{GL}(S) \cdot \mathrm{p}}] \stackrel{\beta^{*}}{\longleftrightarrow} \mathbb{C}\left[\mathrm{GL}(S) \times_{R}(\overline{R \cdot \mathrm{p}})\right] \simeq H^{0}(\mathrm{GL}(S) / R, \mathbb{C}[\overline{R \cdot \mathrm{p}}]) \\
& \stackrel{\alpha^{*}}{\longrightarrow} H^{0}\left(\mathrm{GL}(S) / R, \mathbb{C}\left[S^{*} \times \mathcal{X}_{\text {perm }}\right]^{\mathbb{C}^{*}}\right) \text {. }
\end{aligned}
$$




\section{Determination of $\boldsymbol{H}^{0}\left(\mathrm{GL}(S) / R, \mathbb{C}\left[S^{*} \times \boldsymbol{X}_{\text {perm }}\right]^{\mathbb{C}^{*}}\right)$}

We continue to follow the notation from the last section. In particular, $3 \leq n<m$. For any $d \geq 0$, we have the canonical inclusion:

$$
\begin{aligned}
j: H^{0}\left(\mathrm{GL}(S) / R,\left(\mathbb{C}\left[S^{*}\right] \otimes \mathbb{C}^{d}\left[\mathcal{X}_{\text {perm }}\right]\right)^{\mathbb{C}^{*}}\right) \\
\hookrightarrow H^{0}\left(\mathrm{GL}(S) / R,\left(\mathbb{C}\left[S^{*} \backslash S_{1}^{*}\right] \otimes \mathbb{C}^{d}\left[\mathcal{X}_{\text {perm }}\right]\right)^{\mathbb{C}^{*}}\right),
\end{aligned}
$$

where $\mathbb{C}^{d}\left[\mathcal{X}_{\text {perm }}\right]$ denotes the space of degree $d$-homogeneous functions on $\mathcal{X}_{\text {perm }} \subset$ $S^{n}\left(E^{*}\right)$. Thus, $\mathbb{C}^{d}\left[\mathcal{X}_{\text {perm }}\right]$ is a quotient of $S^{d}\left(S^{n}(E)\right)$. In this section, we will determine the image of $j$.

For any $R$-module $M, H^{0}(\mathrm{GL}(S) / R, M)$ can canonically be identified with the space of regular maps

$$
\left\{\phi: \operatorname{GL}(S) \rightarrow M: \phi(\ell r)=r^{-1} \cdot(\phi(\ell)), \text { for all } \ell \in \mathrm{GL}(S), r \in R\right\} .
$$

Thus, by the Peter-Weyl theorem and the Tannaka-Krěn duality (cf. Chapter III in [BD])

$$
\begin{aligned}
& H^{0}(\mathrm{GL}(S) / R, M) \\
& \simeq \bigoplus_{\lambda=\left(\lambda_{1} \geq \cdots \geq \lambda_{n^{2}+1}\right) \in D(\mathrm{GL}(S))} V_{\mathrm{GL}(S)}(\lambda)^{*} \otimes \operatorname{Hom}_{R}\left(V_{\mathrm{GL}(S)}(\lambda)^{*}, M\right) .
\end{aligned}
$$

We will apply this to the cases $M=\left(\mathbb{C}\left[S^{*}\right] \otimes \mathbb{C}^{d}\left[\mathcal{X}_{\text {perm }}\right]\right)^{\mathbb{C}^{*}}$ and $M=\left(\mathbb{C}\left[S^{*} \backslash S_{1}^{*}\right] \otimes\right.$ $\left.\mathbb{C}^{d}\left[\mathcal{X}_{\text {perm }}\right]\right)^{\mathbb{C}^{*}}$.

7.1 Lemma. Take any $\lambda=\left(\lambda_{1} \geq \cdots \geq \lambda_{n^{2}+1}\right) \in D(\mathrm{GL}(S))$ and any $d \geq 0$. Then, the canonical inclusion

$$
\begin{aligned}
& \operatorname{Hom}_{R}\left(V_{\mathrm{GL}(S)}(\lambda)^{*},\left(\mathbb{C}\left[S^{*}\right] \otimes \mathbb{C}^{d}\left[\mathcal{X}_{\text {perm }}\right]\right)^{\mathbb{C}^{*}}\right) \\
& \hookrightarrow \operatorname{Hom}_{R}\left(V_{\mathrm{GL}(S)}(\lambda)^{*},\left(\mathbb{C}\left[S^{*} \backslash S_{1}^{*}\right] \otimes \mathbb{C}^{d}\left[\mathcal{X}_{\text {perm }}\right]\right)^{\mathbb{C}^{*}}\right)
\end{aligned}
$$

is an isomorphism if $\lambda_{1} \leq 0$.

Moreover, if $\lambda_{1}>0$, then the left side is 0 .

Proof. Take $\phi \in \operatorname{Hom}_{R}\left(V_{\mathrm{GL}(S)}(\lambda)^{*},\left(\mathbb{C}\left[S^{*} \backslash S_{1}^{*}\right] \otimes \mathbb{C}^{d}\left[\mathcal{X}_{\text {perm }}\right]\right)^{\mathbb{C}^{*}}\right)$. Let $v_{\lambda}^{*} \in$ $V_{\mathrm{GL}(S)}(\lambda)^{*}$ be the lowest weight vector of weight $-\lambda$. Then, $\phi$ is completely determined by its value on $v_{\lambda}^{*}$. Let

$$
\phi_{1}:=\phi\left(v_{\lambda}^{*}\right):\left(S^{*} \backslash S_{1}^{*}\right) \times \mathcal{X}_{\text {perm }} \rightarrow \mathbb{C}
$$

be the corresponding map. For $z \in \mathbb{C}^{*}$, take the diagonal matrix $\hat{z}=[z, 1, \ldots, 1] \in$ $\mathrm{GL}(S)$ with respect to the basis $\left\{e_{1,1}, e_{i, j}\right\}_{m-n+1 \leq i, j \leq m}$. Then, $\phi\left(\hat{z} v_{\lambda}^{*}\right)=\hat{z} \cdot \phi\left(v_{\lambda}^{*}\right)$, 
i.e., $e^{-\lambda}(\hat{z}) \phi_{1}=\hat{z} \cdot \phi_{1}$. This gives $z^{-\lambda_{1}} \phi_{1}=\hat{z} \cdot \phi_{1}$, i.e.,

$$
\begin{aligned}
z^{-\lambda_{1}} \phi_{1}\left(\left(z_{1,1}, z_{i, j}\right), x\right) & =\phi_{1}\left(\hat{z}^{-1}\left(\left(z_{1,1}, z_{i, j}\right), x\right)\right) \\
& =\phi_{1}\left(\left(z_{1,1}, z_{i, j}\right), x\right),
\end{aligned}
$$

where $\left\{z_{1,1}, z_{i, j}\right\}$ are the coordinates on $S^{*}$ with respect to the basis $\left\{e_{1,1}, e_{i, j}\right\}$ of $S$. Write

$$
\phi_{1}\left(\left(z_{1,1}, z_{i, j}\right), x\right)=\sum_{\ell \in \mathbb{Z}} z_{1,1}^{\ell} P_{\ell}\left(z_{i, j}, x\right)
$$

for some $P_{\ell}\left(z_{i, j}, x\right) \in \mathbb{C}\left[S_{1}^{*}\right] \otimes \mathbb{C}^{d}\left[\mathcal{X}_{\text {perm }}\right]$. Equation (24) gives

$$
z^{-\lambda_{1}} \sum_{\ell \in \mathbb{Z}} z_{1,1}^{\ell} P_{\ell}\left(z_{i, j}, x\right)=\sum_{\ell \in \mathbb{Z}} z^{\ell} z_{1,1}^{\ell} P_{\ell}\left(z_{i, j}, x\right)
$$

for all $z_{1,1}, z \in \mathbb{C}^{*}, z_{i, j} \in \mathbb{C}$ and $x \in \mathcal{X}_{\text {perm }}$. For any $\ell \in \mathbb{Z}$ such that $P_{\ell}\left(z_{i, j}, x\right) \neq 0$ (for some $z_{i, j} \in \mathbb{C}$ and some $x \in \mathcal{X}_{\text {perm }}$ ), from the above equation, we get $z^{-\lambda_{1}}=z^{\ell}$. In particular,

$$
\phi_{1}\left(\left(z_{1,1}, z_{i, j}\right), x\right)=z_{1,1}^{-\lambda_{1}} P_{-\lambda_{1}}\left(z_{i, j}, x\right) .
$$

Thus, if nonzero, $\phi_{1}:\left(S^{*} \backslash S_{1}^{*}\right) \times \mathcal{X}_{\text {perm }} \rightarrow \mathbb{C}$ extends to a morphism $S^{*} \times \mathcal{X}_{\text {perm }} \rightarrow \mathbb{C}$ iff $-\lambda_{1} \geq 0$. This proves the lemma.

As a corollary of the above lemma and the identity (23), we get the following.

7.2 Proposition. For any $d \geq 0$, let

$$
\begin{aligned}
& H^{0}\left(\mathrm{GL}(S) / R,\left(\mathbb{C}\left[S^{*} \backslash S_{1}^{*}\right] \otimes \mathbb{C}^{d}\left[\mathcal{X}_{\text {perm }}\right]\right)^{\mathbb{C}^{*}}\right) \\
& =\bigoplus_{\lambda=\left(\lambda_{1} \geq \cdots \geq \lambda_{n^{2}+1}\right) \in D(\operatorname{GL}(S))} m_{\lambda}(d) V_{\mathrm{GL}(S)}(\lambda)^{*}
\end{aligned}
$$

Then,

$$
\begin{aligned}
& H^{0}\left(\mathrm{GL}(S) / R,\left(\mathbb{C}\left[S^{*}\right] \otimes \mathbb{C}^{d}\left[\mathcal{X}_{\text {perm }}\right]\right)^{\mathbb{C}^{*}}\right) \\
& \quad=\bigoplus_{\lambda=\left(\lambda_{1} \geq \cdots \geq \lambda_{n^{2}+1}\right) \in D(\mathrm{GL}(S)): \lambda_{1} \leq 0} m_{\lambda}(d) V_{\mathrm{GL}(S)}(\lambda)^{*}
\end{aligned}
$$

Define a new action of $R$ on $X_{\text {perm }}$ by

$$
r \odot x=\chi(r)^{n-m} r \cdot x
$$

where $\chi: R \rightarrow \mathbb{C}^{*}$ is the character defined by $\chi(r)=\left(r e_{1,1}\right)_{1,1}$, where $(X)_{1,1}$ is defined in the proof of Proposition 5.5. 
7.3 Lemma. For any $d \geq 0$, there is a canonical isomorphism of $\mathrm{GL}(S)$-modules:

$$
H^{0}\left(\mathrm{GL}(S) / R,\left(\mathbb{C}\left[S^{*} \backslash S_{1}^{*}\right] \otimes \mathbb{C}^{d}\left[\mathcal{X}_{\text {perm }}\right]\right)^{\mathbb{C}^{*}}\right) \simeq H^{0}\left(\mathrm{GL}(S) / L_{R}, \mathbb{C}^{d}\left[\mathcal{X}_{\text {perm }}\right]^{\chi}\right),
$$

where $\mathbb{C}^{d}\left[X_{\text {perm }}\right]^{\chi}$ is the same space as $\mathbb{C}^{d}\left[X_{\text {perm }}\right]$ but the $L_{R}$-module structure on $\mathbb{C}^{d}\left[\mathcal{X}_{\text {perm }}\right]^{\chi}$ is induced from the action $\odot$ of $R$ (in particular, $\left.L_{R}\right)$ on $X_{\text {perm. }}$.

Proof. From the fibration $R / L_{R} \rightarrow \mathrm{GL}(S) / L_{R} \rightarrow \mathrm{GL}(S) / R$, we get

$$
H^{0}\left(\mathrm{GL}(S) / L_{R}, \mathbb{C}^{d}\left[\mathcal{X}_{\text {perm }}\right]^{\chi}\right) \simeq H^{0}\left(\mathrm{GL}(S) / R, \mathbb{C}\left[R / L_{R}\right] \otimes\left(\mathbb{C}^{d}\left[\mathcal{X}_{\text {perm }}\right]^{\chi}\right)\right) .
$$

So, it suffices to define an $R$-module isomorphism

$$
\gamma:\left(\mathbb{C}\left[S^{*} \backslash S_{1}^{*}\right] \otimes \mathbb{C}^{d}\left[\mathcal{X}_{\text {perm }}\right]\right)^{\mathbb{C}^{*}} \rightarrow \mathbb{C}\left[R / L_{R}\right] \otimes\left(\mathbb{C}^{d}\left[\mathcal{X}_{\text {perm }}\right]^{\chi}\right) .
$$

First, define a morphism $\gamma_{1}: R / L_{R} \rightarrow S^{*} \backslash S_{1}^{*}$ by $\left(\gamma_{1}\left(r L_{R}\right)\right)(X)=\chi(r)\left(r^{-1} X\right)_{1,1}$, for $r \in R$ and $X \in S$. Then, $\gamma_{1}$ satisfies:

$$
\gamma_{1}\left(r^{\prime} r L_{R}\right)=\chi\left(r^{\prime}\right) r^{\prime} \cdot \gamma_{1}\left(r L_{R}\right) \quad \text { for any } r, r^{\prime} \in R .
$$

Now, define the morphism

$$
\bar{\gamma}_{1}: R / L_{R} \times\left(\mathcal{X}_{\text {perm }}, \odot\right) \rightarrow\left(\left(S^{*} \backslash S_{1}^{*}\right) \times \mathcal{X}_{\text {perm }}\right) / / \mathbb{C}^{*},\left(r L_{R}, x\right) \mapsto\left[\gamma_{1}\left(r L_{R}\right), x\right],
$$

where $\left(\mathcal{X}_{\text {perm }}, \odot\right)$ denotes the variety $\mathcal{X}_{\text {perm }}$ together with the action $\odot$ of $R$. From (26), it is easy to see that $\bar{\gamma}_{1}$ is an $R$-equivariant morphism. Moreover, it is a biregular isomorphism. (Observe that all the $\mathbb{C}^{*}$-orbits in $\left(S^{*} \backslash S_{1}^{*}\right) \times \mathcal{X}_{\text {perm }}$ are closed and hence $\left(\left(S^{*} \backslash S_{1}^{*}\right) \times \mathcal{X}_{\text {perm }}\right) / / \mathbb{C}^{*}$ is the same as the orbit space $\left(\left(S^{*} \backslash S_{1}^{*}\right) \times \mathcal{X}_{\text {perm }}\right) / \mathbb{C}^{*}$. $)$ Now, $\gamma$ is nothing but the induced map from $\bar{\gamma}_{1}$.

Now, we determine $H^{0}\left(\mathrm{GL}(S) / L_{R}, \mathbb{C}^{d}\left[\mathcal{X}_{\text {perm }}\right]^{\chi}\right)$.

7.4 Lemma. For any $d \geq 0$,

$$
\begin{aligned}
& H^{0}\left(\mathrm{GL}(S) / L_{R}, \mathbb{C}^{d}\left[\mathcal{X}_{\text {perm }}\right]^{\chi}\right) \\
& \simeq \bigoplus_{\lambda=\left(\lambda_{1} \geq \cdots \geq \lambda_{n^{2}+1}\right) \in D(\mathrm{GL}(S))} V_{\mathrm{GL}(S)}(\lambda) \otimes \operatorname{Hom}_{L_{R}}\left(V_{\mathrm{GL}(S)}(\lambda), \mathbb{C}^{d}\left[X_{\text {perm }}\right]^{\chi}\right) .
\end{aligned}
$$

Thus, for any $\lambda=\left(\lambda_{1} \geq \lambda_{2} \geq \cdots \geq \lambda_{n^{2}+1}\right) \in D(\mathrm{GL}(S)), V_{\mathrm{GL}(S)}(\lambda)$ appears in $H^{0}\left(\mathrm{GL}(S) / L_{R}, \mathbb{C}^{d}\left[\mathcal{X}_{\text {perm }}\right]^{\chi}\right)$ if and only if the following two conditions are satisfied:

(1) $|\lambda|=d m$, where $|\lambda|:=\sum \lambda_{i}$, and

(2) there exists $\mu=\left(\mu_{1} \geq \cdots \geq \mu_{n^{2}}\right)$ such that $\mu$ interlaces $\lambda$, i.e.,

$$
\lambda_{1} \geq \mu_{1} \geq \lambda_{2} \geq \mu_{2} \geq \cdots \geq \lambda_{n^{2}} \geq \mu_{n^{2}} \geq \lambda_{n^{2}+1},
$$

and the $\mathrm{GL}\left(S_{1}\right)$-irreducible module $V_{\mathrm{GL}\left(S_{1}\right)}(\mu)$ appears in $\mathbb{C}^{d}\left[\mathcal{X}_{\text {perm }}\right]$. 
Proof. The isomorphism (27) of course follows from the Peter-Weyl theorem and the Tannaka-Kreǐn duality.

For $z \in \mathbb{C}^{*}$, let $\bar{z}$ be the diagonal matrix $[1, z, \ldots, z] \in$ Aut $S_{1} \subset$ Aut $S$ and $\hat{z}$ the diagonal matrix $[z, 1, \ldots, 1] \in \operatorname{Aut}\left(\mathbb{C} e_{1,1}\right) \subset$ Aut $S$. Then, $\bar{z} \hat{z}$ acts on $\mathcal{X}_{\text {perm }}$ via

$$
(\bar{z} \hat{z}) \odot x=z^{n-m}(\bar{z} \cdot x)=z^{-m} x .
$$

By the branching law for the pair $\left(\mathrm{GL}(S), \mathrm{GL}\left(S_{1}\right)\right)$ (cf. [GW], Theorem 8.1.1), we get, for any $\lambda \in D(\mathrm{GL}(S))$,

$$
V_{\mathrm{GL}(S)}(\lambda) \simeq \bigoplus_{\substack{\mu \in D\left(\mathrm{GL}\left(S_{1}\right)\right): \\ \mu \text { interlaces } \lambda}} V_{\mathrm{GL}\left(S_{1}\right)}(\mu), \text { as } \operatorname{GL}\left(S_{1}\right) \text {-modules. }
$$

Now, since $\operatorname{GL}\left(S_{1}\right)$ and $\bar{z} \hat{z}$ generate the group $L_{R}$, combining the equations (27)(29), we get the second part of the lemma. (Observe that the two actions - and $\odot$ of $\operatorname{GL}\left(S_{1}\right)$ on $\mathcal{X}_{\text {perm }}$ coincide. $)$

Combining Proposition 7.2 with the Lemmas 7.3-7.4 and the identities (28)-(29), we get the following:

7.5 Theorem. For any $d \geq 0$, decompose

$$
\mathbb{C}^{d}\left[\mathcal{X}_{\text {perm }}\right] \simeq \oplus_{\mu \in D\left(\mathrm{GL}\left(S_{1}\right)\right)} q_{\mu}(d) V_{\mathrm{GL}\left(S_{1}\right)}(\mu)
$$

as $\mathrm{GL}\left(S_{1}\right)$-modules. Then, as $\mathrm{GL}(S)$-modules,

$$
\begin{aligned}
& H^{0}\left(\mathrm{GL}(S) / R,\left(\mathbb{C}\left[S^{*}\right] \otimes \mathbb{C}^{d}\left[\mathcal{X}_{\text {perm }}\right]\right)^{\mathbb{C}^{*}}\right)
\end{aligned}
$$

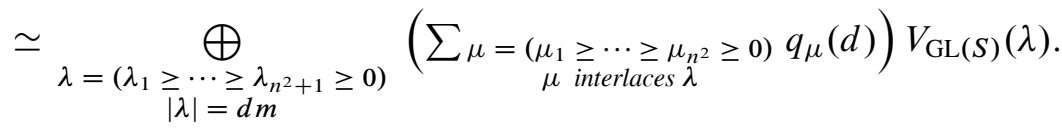

In particular, $V_{\mathrm{GL}(S)}(\lambda)$ occurs in $H^{0}\left(\mathrm{GL}(S) / R,\left(\mathbb{C}\left[S^{*}\right] \otimes \mathbb{C}^{d}\left[\mathcal{X}_{\text {perm }}\right]\right)^{\mathbb{C}^{*}}\right)$ if and only if the following two conditions are satisfied:

(1) $\lambda=\left(\lambda_{1} \geq \cdots \geq \lambda_{n^{2}+1} \geq 0\right)$ and $|\lambda|=d m$, and

(2) there exists a $\mu=\left(\mu_{1} \geq \cdots \geq \mu_{n^{2}} \geq 0\right)$ which interlaces $\lambda$ and such that the irreducible $G L\left(S_{1}\right)$-module $\bar{V}_{\mathrm{GL}\left(S_{1}\right)}(\mu)$ occurs in $\mathbb{C}^{d}\left[\mathcal{X}_{\text {perm }}\right]$.

(Observe that if $V_{\mathrm{GL}\left(S_{1}\right)}(\mu)$ occurs in $\mathbb{C}^{d}\left[\mathcal{X}_{\text {perm }}\right]$, then automatically $|\mu|=d n$ and $\mu_{n^{2}} \geq 0$, since $\mathbb{C}^{d}\left[\mathcal{X}_{\text {perm }}\right]$ is a $\operatorname{GL}\left(S_{1}\right)$-module quotient of $S^{d}\left(S^{n}(E)\right)$.)

7.6 Remark. Since

$$
\left(\mathbb{C}\left[S^{*}\right] \otimes \mathbb{C}^{d}\left[\mathcal{X}_{\text {perm }}\right]\right)^{\mathbb{C}^{*}} \simeq S^{(m-n) d}(S) \otimes \mathbb{C}^{d}\left[\mathcal{X}_{\text {perm }}\right],
$$


and $S$ is a GL( $S)$-module, we also get (using [Ku1], Lemma 8)

$$
\begin{aligned}
& H^{0}\left(\mathrm{GL}(S) / R,\left(\mathbb{C}\left[S^{*}\right] \otimes \mathbb{C}^{d}\left[\mathcal{X}_{\text {perm }}\right]\right)^{\mathbb{C}^{*}}\right) \\
& \quad \simeq S^{(m-n) d}(S) \otimes H^{0}\left(\mathrm{GL}(S) / R, \mathbb{C}^{d}\left[\mathcal{X}_{\text {perm }}\right]\right) \\
& \quad \simeq \bigoplus_{\mu=\left(\mu_{1} \geq \cdots \geq \mu_{n^{2}}\right): \mu_{n^{2}} \geq 0} q_{\mu}(d) S^{(m-n) d}(S) \otimes V_{\mathrm{GL}(S)}(\hat{\mu}),
\end{aligned}
$$

where $\hat{\mu}:=\left(\mu_{1} \geq \cdots \geq \mu_{n^{2}} \geq 0\right) \in D(\operatorname{GL}(S))$.

\section{Nonnormality of the orbit closures of $p$}

It is easy to see that the morphism $\alpha$ of Section 6 induces an injective map (for any $d \geq 0$ )

$$
\alpha^{*}: \mathbb{C}^{d}[\overline{R \cdot \mathrm{p}}] \hookrightarrow\left(\mathbb{C}\left[S^{*}\right] \otimes \mathbb{C}^{d}\left[\mathcal{X}_{\text {perm }}\right]\right)^{\mathbb{C}^{*}}=S^{d(m-n)}(S) \otimes \mathbb{C}^{d}\left[\mathcal{X}_{\text {perm }}\right] .
$$

8.1 Proposition. For any $m \geq 2 n$, the inclusion

$$
H^{0}\left(\mathrm{GL}(S) / R, \mathbb{C}^{d}[\overline{R \cdot \mathrm{p}}]\right) \hookrightarrow H^{0}\left(\mathrm{GL}(S) / R,\left(\mathbb{C}\left[S^{*}\right] \otimes \mathbb{C}^{d}\left[\mathcal{X}_{\text {perm }}\right]\right)^{\mathbb{C}^{*}}\right),
$$

induced from the inclusion $\alpha^{*}$, is not an isomorphism for $d=1$.

Proof. Of course, $\mathbb{C}^{1}\left[\overline{R \cdot p]}\right.$ is a $R$-module quotient of $S^{m}(E)$; in fact, it is a $R$-module quotient of $S^{m}(S)$. Let $K$ be the kernel

$$
0 \rightarrow K \rightarrow S^{m}(S) \rightarrow \mathbb{C}^{1}[\overline{R \cdot \mathrm{p}}] \rightarrow 0 .
$$

We first determine the linear span $\langle\overline{R \cdot \mathrm{p}}\rangle$ of the image of $\overline{R \cdot \mathrm{p}}$ inside $S^{m}\left(S^{*}\right)$. For $u \in U_{R}, z \in \mathbb{C}^{*}$ and $g \in \operatorname{GL}\left(S_{1}\right)$ (where $\tau_{z} \in \operatorname{Aut}\left(\mathbb{C} e_{1,1}\right)$ is defined by $\left.\tau_{z}\left(e_{1,1}\right)=z e_{1,1}\right)$,

$$
\begin{aligned}
&\left(\left(g u \tau_{z}\right)^{-1} \cdot \mathrm{p}\right)\left(x_{1,1} e_{1,1}+\sum_{m-n+1 \leq i, j \leq m} x_{i, j} e_{i, j}\right) \\
&= \mathrm{p}\left(\left(z x_{1,1}+\sum x_{i, j} a_{i, j}\right) e_{1,1}+g \sum x_{i, j} e_{i, j}\right) \\
& \quad\left(\text { where } u e_{i, j}=e_{i, j}+a_{i, j} e_{1,1}\right) \\
&=\left(z x_{1,1}+\sum x_{i, j} a_{i, j}\right)^{m-n}\left(g^{-1} \cdot \operatorname{perm}\right)\left(\sum x_{i, j} e_{i, j}\right) .
\end{aligned}
$$

For any vector space $V$, the span of $\left\{v^{m-n}, v \in V\right\}$ inside $S^{m-n}(V)$ coincides with $S^{m-n}(V)$. Furthermore, since $S^{n}\left(S_{1}^{*}\right)$ is an irreducible GL $\left(S_{1}\right)$-module, the span of $\left\{g^{-1} \cdot \operatorname{perm}\right\}_{g \in \mathrm{GL}\left(S_{1}\right)}$ is equal to $S^{n}\left(S_{1}^{*}\right)$. Here we have identified $S^{n}\left(S_{1}^{*}\right) \hookrightarrow S^{n}\left(S^{*}\right)$ via the projection $S \rightarrow S_{1}, e_{1,1} \mapsto 0$. 
Thus,

$$
\begin{aligned}
\langle\overline{R \cdot \mathrm{p}}\rangle= & S^{n}\left(S_{1}^{*}\right) \cdot S^{m-n}\left(S^{*}\right) \\
= & \lambda_{o}^{m-n} S^{n}\left(S_{1}^{*}\right) \oplus \lambda_{o}^{m-n-1} S^{n+1}\left(S_{1}^{*}\right) \\
& \oplus \cdots \oplus \lambda_{o}^{0} S^{m}\left(S_{1}^{*}\right),
\end{aligned}
$$

where $\lambda_{o} \in S^{*}$ is defined in the proof of Proposition 6.3. Thus,

$$
K \simeq e_{1,1}^{m-n+1} S^{n-1}\left(S_{1}\right) \oplus \cdots \oplus e_{1,1}^{m} S^{0}\left(S_{1}\right)
$$

None of the weights of $K$ are $\operatorname{GL}(S)$-antidominant with respect to the basis $\left\{e_{1,1}, e_{i, j}\right\}_{m-n+1 \leq i, j \leq m}$ of $S$ if

$$
m-n+1>n-1, \quad \text { i.e., if } m>2 n-2 .
$$

Hence,

$$
H^{0}(\mathrm{GL}(S) / R, K)=0 \text { if } m>2 n-2 \text {. }
$$

Also,

$$
H^{1}(\mathrm{GL}(S) / R, K)=0 \quad \text { if } m>2 n-1 .
$$

To prove this, it suffices to show that, for any weight $\mu$ of $K$ and any simple reflection $s_{i}$ for $\operatorname{GL}(S), s_{i}(-\mu+\rho)-\rho$ is not dominant, i.e., $s_{i} \mu+\alpha_{i}$ is not antidominant. Writing $\mu=\left(\mu_{1}, \ldots, \mu_{n^{2}+1}\right)$, we have

$$
\mu_{1}>\mu_{j}+1 \text { for all } j \geq 2(\text { since } m>2 n-1) .
$$

Thus, if $i>1$,

$$
\left(s_{i} \mu+\alpha_{i}\right)_{1}=\mu_{1}>\left(s_{i} \mu+\alpha_{i}\right)_{2} .
$$

Hence, $s_{i} \mu+\alpha_{i}$ is not antidominant for $i>1$. For $i=1$, we get

$$
\left(s_{1} \mu+\alpha_{1}\right)_{2}=\mu_{1}-1>\left(s_{1} \mu+\alpha_{1}\right)_{3}=\mu_{3} .
$$

Combining (32)-(33), we get

$$
H^{0}(\mathrm{GL}(S) / R, K)=H^{1}(\mathrm{GL}(S) / R, K)=0 \text { for all } m \geq 2 n \text {. }
$$

Considering the long exact cohomology sequence, corresponding to the coefficient sequence (31), we get for all $m \geq 2 n$ (by using (34)),

$$
H^{0}\left(\mathrm{GL}(S) / R, \mathbb{C}^{1}[\overline{R \cdot \mathrm{p}}]\right) \simeq H^{0}\left(\mathrm{GL}(S) / R, S^{m}(S)\right)=S^{m}(S) .
$$

In particular, $H^{0}\left(\mathrm{GL}(S) / R, \mathbb{C}^{1}[\overline{R \cdot \mathrm{p}}]\right)$ is an irreducible $\mathrm{GL}(S)$-module.

Next, we determine $M:=H^{0}\left(\mathrm{GL}(S) / R,\left(\mathbb{C}\left[S^{*}\right] \otimes \mathbb{C}^{1}\left[\mathcal{X}_{\text {perm }}\right]\right)^{\mathbb{C}^{*}}\right)$. (In fact, for the following determination of $M$, we only require $m>n \geq 3$.) By Theorem 7.5, 
the irreducible GL(S)-module $V_{\mathrm{GL}(S)}(\lambda)$ appears in $M$ if and only if the following three conditions are satisfied:

1) $\lambda_{n^{2}+1} \geq 0, \quad|\lambda|=m$,

2) there exists $\mu=\left(\mu_{1} \geq \cdots \geq \mu_{n^{2}} \geq 0\right)$ which interlaces $\lambda$, and

$3)$ the irreducible $\mathrm{GL}\left(S_{1}\right)$-module $V_{\mathrm{GL}\left(S_{1}\right)}(\mu)$ occurs in $\mathbb{C}^{1}\left[\mathcal{X}_{\text {perm }}\right]$.

But, $\mathbb{C}^{1}\left[\mathcal{X}_{\text {perm }}\right]$ is the irreducible $\operatorname{GL}\left(S_{1}\right)$-module $S^{n}\left(S_{1}\right)$, since $\mathcal{X}_{\text {perm }}$ is a closed $\mathrm{GL}\left(S_{1}\right)$-subvariety of $S^{n}\left(S_{1}^{*}\right)$. Thus, $\mu=(n \geq 0 \geq 0 \geq \cdots \geq 0)$. Hence, $V_{\mathrm{GL}(S)}(\lambda)$ occurs in $M$ if and only if

$$
\lambda=\left(\lambda_{1} \geq \lambda_{2} \geq 0 \cdots \geq 0\right) \quad \text { with } \lambda_{1} \geq n \geq \lambda_{2} \text { and } \lambda_{1}+\lambda_{2}=m .
$$

In particular, $M$ is not irreducible. This proves the proposition.

8.2 Corollary. Let $m \geq 2 n$. Then, $\overline{R \cdot p}$ is not normal.

Proof. If $\overline{R \cdot p}$ were normal, by the original form of the Zariski's main theorem (cf. [M], Chapter III, §9) and Proposition 6.3 (following its notation),

$$
\alpha^{*}: \mathbb{C}[\overline{R \cdot \mathrm{p}}] \rightarrow \mathbb{C}\left[\left(S^{*} \times \mathcal{X}_{\text {perm }}\right) / / \mathbb{C}^{*}\right]
$$

would be an isomorphism. In particular, we would get the $R$-module isomorphism

$$
\alpha^{*}: \mathbb{C}^{1}[\overline{R \cdot \mathrm{p}}] \stackrel{\sim}{\longrightarrow}\left(\mathbb{C}\left[S^{*}\right] \otimes \mathbb{C}^{1}\left[\mathcal{X}_{\text {perm }}\right]\right)^{\mathbb{C}^{*}} .
$$

But this contradicts Proposition 8.1.

The following corollary follows similarly.

8.3 Corollary. Let $m \geq 2 n$. Then, $\overline{\mathrm{GL}(S) \cdot \mathrm{p}}$ is not normal.

Proof. By Definition 6.1 and Lemma 6.2, we have the proper, surjective, birational morphism

$$
\beta: \operatorname{GL}(S) \times_{R}(\overline{R \cdot p}) \rightarrow \overline{\mathrm{GL}(S) \cdot \mathrm{p}} .
$$

If $\overline{\mathrm{GL}(S) \cdot \mathrm{p}}$ were normal, both the maps $\beta$ and the composite map $\beta \circ(\operatorname{Id} \times \alpha)$ (which are both proper and birational morphisms)

$$
\mathrm{GL}(S) \times_{R}\left(\left(S^{*} \times \mathcal{X}_{\text {perm }}\right) / / \mathbb{C}^{*}\right) \stackrel{\mathrm{Id} \times \alpha}{\longrightarrow} \mathrm{GL}(S) \times_{R}(\overline{R \cdot \mathrm{p}}) \stackrel{\beta}{\rightarrow} \overline{\mathrm{GL}(S) \cdot \mathrm{p}}
$$

would induce isomorphisms (via the Zariski's main theorem [H], Chapter III, Corollary 11.4 and its proof)

$$
\beta^{*}: \mathbb{C}[\overline{\mathrm{GL}(S) \cdot \mathrm{p}}] \stackrel{\sim}{\longrightarrow} H^{0}(\mathrm{GL}(S) / R, \mathbb{C}[\overline{R \cdot \mathrm{p}}])
$$


and

$$
(\beta \circ(\operatorname{Id} \times \alpha))^{*}: \mathbb{C}[\overline{\mathrm{GL}(S) \cdot \mathrm{p}}] \stackrel{\sim}{\longrightarrow} H^{0}\left(\mathrm{GL}(S) / R, \mathbb{C}\left[S^{*} \times X_{\text {perm }}\right]^{\mathbb{C}^{*}}\right) .
$$

In particular, the canonical map

$$
(\mathrm{Id} \times \alpha)^{*}: H^{0}(\mathrm{GL}(S) / R, \mathbb{C}[\overline{R \cdot \mathrm{p}}]) \stackrel{\sim}{\longrightarrow} H^{0}\left(\mathrm{GL}(S) / R, \mathbb{C}\left[S^{*} \times \mathcal{X}_{\text {perm }}\right]^{\mathbb{C}^{*}}\right)
$$

would be an isomorphism. This contradicts Proposition 8.1. Hence $\overline{\operatorname{GL}(S) \cdot \mathrm{p}}$ is not normal.

8.4 Theorem. Let $m>n \geq 3$. Then, $\overline{G \cdot \mathrm{p}}$ is not normal.

Proof. Recall from Section 5 the proper and surjective morphism $\phi: G \times{ }_{P}(\overline{P \cdot p)} \rightarrow$ $\overline{G \cdot \mathrm{p}}$. It is birational by Corollary 5.4. Consider the projection $\pi: P \rightarrow \operatorname{GL}(S)$, obtained by identifying $\operatorname{GL}(S) \simeq P /\left(U_{P} \cdot \mathrm{GL}\left(S^{\perp}\right)\right)$ and let $P_{R}$ be the parabolic subgroup of $P$ defined as $\pi^{-1}(R)$. Now, define the variety

$$
Y=P \times_{P_{R}}\left(\left(S^{*} \times X_{\text {perm }}\right) / / \mathbb{C}^{*}\right),
$$

where $P_{R}$ acts on $\left(S^{*} \times \mathcal{X}_{\text {perm }}\right) / / \mathbb{C}^{*}$ via its projection onto $R$. Consider the morphism

$$
\alpha_{P}: Y \rightarrow \overline{P \cdot \mathrm{p}}=\overline{\mathrm{GL}(S) \cdot \mathrm{p}}, \quad[p, x] \mapsto p \cdot \alpha(x),
$$

for $p \in P$ and $x \in\left(S^{*} \times \mathcal{X}_{\text {perm }}\right) / / \mathbb{C}^{*}$. Observe that, under the canonical identification (induced from the map $\pi) \operatorname{GL}(S) \times_{R}\left(\left(S^{*} \times \mathcal{X}_{\text {perm }}\right) / / \mathbb{C}^{*}\right) \simeq Y$, the map $\alpha_{P}$ is nothing but the composite map $\beta \circ(\operatorname{Id} \times \alpha)$ (cf., the proof of Corollary 8.3). Hence, $\alpha_{P}$ is a proper, birational morphism. The $P$-morphism $\alpha_{P}$ of course gives rise to a proper, birational $G$-morphism

$$
\bar{\alpha}_{P}: G \times_{P} Y \rightarrow G \times_{P}(\overline{P \cdot \mathrm{p}}) .
$$

Finally, define the proper, birational, surjective $G$-morphism as the composite

$$
\hat{\alpha}_{P}:=\phi \circ \bar{\alpha}_{P}: G \times_{P} Y \rightarrow \overline{G \cdot \mathrm{p}}
$$

If $\overline{G \cdot p}$ were normal, we would get an isomorphism

$$
\hat{\alpha}_{P}{ }^{*}: \mathbb{C}[\overline{G \cdot \mathrm{p}}] \rightarrow \mathbb{C}\left[G \times_{P} Y\right] \simeq H^{0}\left(G / P, H^{0}\left(\mathrm{GL}(S) / R, \mathbb{C}\left[S^{*} \times \mathcal{X}_{\text {perm }}\right]^{\mathbb{C}^{*}}\right)\right),
$$

where $P$ acts on $H^{0}\left(\mathrm{GL}(S) / R, \mathbb{C}\left[S^{*} \times \mathcal{X}_{\text {perm }}\right]^{\mathbb{C}^{*}}\right)$ via its projection $\pi$. It is easy to see that this, in particular, would induce an isomorphism

$$
\mathbb{C}^{1}[\overline{G \cdot \mathrm{p}}] \simeq H^{0}\left(G / P, H^{0}\left(\mathrm{GL}(S) / R,\left(\mathbb{C}\left[S^{*}\right] \otimes \mathbb{C}^{1}\left[\mathcal{X}_{\text {perm }}\right]\right)^{\mathbb{C}^{*}}\right)\right) .
$$


Now, by the proof of Proposition 8.1 (this part being valid under the only assumption $m>n \geq 3$ ), there exist $k_{\lambda}>0$ such that

$$
\begin{aligned}
& H^{0}\left(G / P, H^{0}\left(\mathrm{GL}(S) / R,\left(\mathbb{C}\left[S^{*}\right] \otimes \mathbb{C}^{1}\left[\chi_{\text {perm }}\right]\right)^{\mathbb{C}^{*}}\right)\right) \\
& \simeq \bigoplus_{\lambda=\left(\lambda_{1} \geq \lambda_{2} \geq 0 \geq \cdots \geq 0\right) \in D(\mathrm{GL}(S)): \lambda_{1} \geq n \geq \lambda_{2}, \lambda_{1}+\lambda_{2}=m} k_{\lambda} H^{0}\left(G / P, V_{\mathrm{GL}(S)}(\lambda)\right) \\
& \simeq \bigoplus_{\hat{\lambda}=\left(\lambda_{1} \geq \lambda_{2} \geq 0 \geq \cdots \geq 0\right) \in D(G): \lambda_{1} \geq n \geq \lambda_{2}, \lambda_{1}+\lambda_{2}=m} k_{\lambda} V_{G}(\hat{\lambda}), \quad \text { by }[\operatorname{Ku} 1], \text { Lemma } 8,
\end{aligned}
$$

where $\hat{\lambda}$ is obtained from $\lambda$ by adding $m^{2}-n^{2}-1$ zeroes in the end to $\lambda$. In particular, $H^{0}\left(G / P, H^{0}\left(\mathrm{GL}(S) / R,\left(\mathbb{C}\left[S^{*}\right] \otimes \mathbb{C}^{1}\left[\mathcal{X}_{\text {perm }}\right]\right)^{\mathbb{C}^{*}}\right)\right)$ is not an irreducible $G$-module.

Finally, $\mathbb{C}^{1}[\overline{G \cdot \mathrm{p}}]$ is, by definition, a $G$-module quotient of the irreducible $G$ module $Q^{*} \simeq S^{m}(E)$. Clearly, $\mathbb{C}^{1}[\overline{G \cdot \mathrm{p}}]$ is nonzero and hence

$$
\mathbb{C}^{1}[\overline{G \cdot \mathrm{p}}] \simeq S^{m}(E) .
$$

This contradicts (36) and hence the theorem is proved.

8.5 Remark. (a) As pointed out by N. Bushek, it is easy to see (by using that $\phi^{*}$ is an isomorphism as in Theorem 5.2, and considering the normalization of $\overline{G \cdot p}$ ) that if $\overline{\mathrm{GL}}(S) \cdot \mathrm{p}$ is normal, then so is $\overline{G \cdot \mathrm{p}}$. Thus, using Theorem 8.4 , we get that $\overline{\operatorname{GL}(S) \cdot \mathrm{p}}$ is not normal for any $m>n \geq 3$ (thereby improving Corollary 8.3).

(b) I thank Bushek for pointing out that the hypothesis $m \geq 2 n$ in Theorem 8.4 in an earlier draft of the paper was unnecessary (with no change in the proof).

(c) Corollary 8.2 holds for any $m>n \geq 3$. To prove it for $3 \leq n<m<2 n$, it is easy to see, from the proof of Proposition 8.1 , that $\operatorname{dim} \mathbb{C}^{1}[\overline{R \cdot \mathrm{p}}]<\operatorname{dim}\left(\mathbb{C}\left[S^{*}\right] \otimes\right.$ $\left.\mathbb{C}^{1}\left[\mathcal{X}_{\text {perm }}\right]\right)^{\mathbb{C}^{*}}$

\section{References}

[AM] M. Atiyah and I. Macdonald, Introduction to commutative algebra. Addison-Wesley Publishing Company, Reading, Mass., 1969. Zbl 0175.03601 MR 0242802

[BL] P. Bürgisser, J. Landsberg, L. Manivel, and J. Weyman, An overview of mathematical issues arising in the geometric complexity theory approach to VP $\neq$ VNP. SIAM J. Comput. 40 (2011), no. 4, 1179-1209. Zbl 1252.68134 MR 2861717

[B] P. Botta, Linear transformations that preserve the permanent. Proc. Amer. Math. Soc. 18 (1967), 566-569. Zbl 0148.25704 MR 0213376

[BD] T. Bröcker and T. tom Dieck, Representations of compact Lie groups. Grad. Texts in Math. 98. Springer-Verlag, New York 1985. Zbl 0581.22009 MR 781344

[Bo] N. Bourbaki, Éléments de mathématique. Groupes et Algèbres de Lie. Chapitres 4-6. Masson, Paris 1981. Zbl 0483.22001 MR 0647314

[Fr] G. Frobenius, Über die Darstellung der endlichen Gruppen durch lineare Substitutionen. Sitzungsber. Preuss. Akad. Wiss. Berlin (1897), 994-1015. JFM 28.0130.01 
[F] W. Fulton, Young tableaux. London Math. Soc. Stud. Texts 35, Cambridge University Press, Cambridge 1997. Zbl 0878.14034 MR 1464693

[GW] R. Goodman and N. Wallach, Symmetry, representations, and invariants. Grad. Texts in Math. 255, Springer-Verlag, Dordrecht 2009. Zbl 1173.22001 MR 2522486

[GM] A. Guterman and A. Mikhalev, General algebra and linear transformations preserving matrix invariants. J. Math. Sci. 128 (2005), 3384-3395. Zbl 1073.15004 MR 2072621

[H] R. Hartshorne, Algebraic geometry. Grad. Texts in Math. 52, Springer-Verlag, Heidelberg 1977. Zbl 0367.14001 MR 0463157

[Ho] R. Howe, $\left(\mathrm{GL}_{n}, \mathrm{GL}_{m}\right)$-duality and symmetric plethysm. Proc. Indian Acad. Sci. (Math. Sci.) 97 (1987), 85-109. Zbl 0705.20040 MR 0983608

[Ke] G. Kempf, Instability in invariant theory. Ann. of Math. 108 (1978), 299-316. Zbl 0406.14031 MR 0506989

[Ku1] S. Kumar, Symmetric and exterior powers of homogeneous vector bundles. Math. Ann. 299 (1994), 293-298. Zbl 0811.20043 MR 1275769

[Ku2] S. Kumar, Kac-Moody groups, their flag varieties and representation theory. Progr. Math. 204, Birkhäuser, Boston, Mass., 2002. Zbl 1026.17030 MR 1923198

[LMR] J. Landsberg, L. Manivel, and N. Ressayre, Hypersurfaces with degenerate duals and the geometric complexity theory program. Comment. Math. Helv. 88 (2013), no. 2, 469-484. Zbl 06176455 MR 3048194

[MM] M. Marcus and F. May, The permanent function. Canadian J. of Math. 14 (1962), 177-189. Zbl 0106.01601 MR 0137729

[MS1] K. Mulmuley and M. Sohoni, Geometric complexity theory I: An approach to the P vs. NP and related problems. SIAM J. Comput. 31 (2001), 496-526. Zbl 0992.03048 MR 1861288

[MS2] K. Mulmuley and M. Sohoni, Geometric complexity theory II: Towards explicit obstructions for embeddings among class varieties. SIAM J. Comput. 38 (2008), 1175-1206. Zbl 1168.03030 MR 2421083

[M] D. Mumford, The red book of varieties and schemes. Lecture Notes in Math. 1358, Springer-Verlag, Berlin 1988. Zbl 0658.14001 MR 0971985

[S] I. Shafarevich, Basic algebraic geometry 1. Springer-Verlag, Berlin 1994. Zbl 0797.14001 MR 1328833

[V] L. G. Valiant, Completeness classes in algebra. In Proceedings of the Eleventh Annual ACM Symposium on Theory of Computing (Atlanta, Ga., 1979), ACM, New York 1979, 249-261. MR 0564634

Received August 3, 2010

Shrawan Kumar, Department of Mathematics, University of North Carolina, Chapel Hill, NC 27599-3250, U.S.A.

E-mail: shrawan@email.unc.edu 